\title{
Effect of mineral and organic fertilizer rates on melon vegetative growth and its productivity
}

\author{
Merghany, M.M ${ }^{1}$, Amal M. Farag ${ }^{1}$, Emam, Y.T $^{2}$. and Ghada A. Tawfic ${ }^{1}$ \\ 1-Vegetable Crops Department, Faculty of Agriculture, Cairo University \\ 2-Horticulture Research Institute, Agric.Res.Center
}

\begin{abstract}
Two field experiments were carried out at the farm of Agriculture Researches Station, Fac. Agric, Cairo Univ., Giza during two successive summer seasons of 2011 and 2012 aiming to investigate the effect of organic and inorganic fertilizers and their interaction on melon vegetative growth and its yield. The obtained results cleared that applying 2 ton compost $+60 \mathrm{~kg} \mathrm{~N} /$ fed enhanced plant length, number of leaves, plant fresh and dry weight, number of fruits, average fruit weight and yield per plant and per feddan in both seasons. The highest values of vegetative growth characters, average fruit weight, number of fruits and yield were recorded with 150 $\mathrm{kg} \mathrm{K} 2 \mathrm{O}$ /fed compared to the other treatment of $100 \mathrm{~kg} \mathrm{~K}_{2} \mathrm{O} / \mathrm{fed}$ in both seasons. No significant differences were recorded on vegetative growth character (plant length and number of leaves) due to the application of foliar calcium treatment in both seasons. But there was a significant effect on plant fresh and dry weigh, average fruit weight, number of fruits and yield due to calcium treatment. The interactions between treatments in this study have been discussed.
\end{abstract}

Keywords: melon (Cucumis melo), mineral, organic and inorganic fertilizers, vegetative growth, yield.

\section{Introduction}

Melon (Cucumis melo .) is one of the most vegetable crops in Egypt for local consumption and exportations. Melon area was 643067 feddan that produced 11.3 ton per fed 2010 season. (Ministry of Agric, Egypt 2011). Commercial and subsistence farming has been and is still relying on the use of inorganic fertilizers for growing crops (Lampkin, 1990), this is because they are easy to use, quickly absorbed and utilized by crops. However, these fertilizers are believed to contribute substantially to human, animal food intoxication and environmental instability/degradation.

The chemical fertilizers used in conventional agriculture contain just a few minerals, which dissolve quickly in damp soil and give the plants large doses of minerals (Vernon, 1999). Although chemical fertilizers have been claimed as the most important contributor to the increase in world agricultural productivity over the past decades (Smil, 2001) The negative effects of chemical fertilizer on soil and environment limit its usage in sustainable agricultural systems (Peyvast et al., 2008). Weakening soil quality requires increasing inputs to maintain high yields. This, in turn, threatens future food security and raises production costs for often already poor farmers

Organic fertilizers can therefore be used to reduce the amount of toxic compounds (such as nitrates) produced by conventional fertilizers in vegetables.

Increased consumer awareness of food safety issues and environmental concerns has contributed to the development of organic farming over the last few years (Worthington, 1998 and 2001; Relf et al., 2002). Thus, it may be possible to lessen the escalating effects of diseases such as cancer and boost immunity of humans. Farming come will also improve when farmers use less money on fertilizers and pesticides for growing crops, (Vernon, 1999).

The use of organic manure has been the need of by production input for improving the sustainable productivity of soil. Addition of compost improves soil structure, texture and tilth (Hesse and Mishra, 1982).

Research comparing soils of organically and chemically managed farming systems has recognized the higher soil organic matter and total nitrogen $(\mathrm{N})$ with the use of organic agriculture (Alvarez et al., 1988; Drinkwater et al., 1995; Reganold, 1988). Soil $\mathrm{pH}$ becomes higher, plant-available nutrient concentrations may be higher, and the total microbial population increases under organic management (Clark et al., 1998; Dinesh et al., 2000; Reganold, 1988; Lee, 2010)

The combination of organic materials with reduced NPK fertilizer rates produced plants that were similar to unmixed NPK fertilizer. This indicated that the high dose of organic manures can be reduced by half and mixed with a reduced rate of NPK fertilizers as reported by Akande et al. (2003).

Plant length, number of leaves, fresh and dry weight were increased when organic and mineral fertilizer combined together (Farrag, 2009 on cantaloupe; olaniyi et al, 2009 and Eifediyi and Remison , 2010 on cucumber ;Olaniyi and Odedere, 2009 on fluted pumpkin.

Plant length, number of leaves ,fresh and dry weight were increased by increasing $\mathrm{N}$ mineral fertilizer( Shebl ,1995 Adam et al,2002 on cantaloupe.; Castellanos , 2011on melon and Abou El-yaized 2012 on cantaloupe ; Jilani et al, 2009 on 
cucumber ).

Thus, the aim of this study was to investigate the effect of the combination between organic and mineral fertilization on growth, yield and its component of melon.

\section{Materials and methods}

This investigation was carried at the farm of Agric. Res. Station, Fac. Agric. Cairo University, Egypt during the two successive summer seasons of 2011 and 2012 to study the effect of organic and mineral fertilization on growth, yield and its components. Table 1 and 2 show the physical and chemical properties of the experimental soil and compost chemical characteristics according to Piper (1950)

Table 1. Some physical and chemical properties of soil

\begin{tabular}{|c|c|c|c|c|c|c|c|c|c|c|c|c|c|c|c|c|}
\hline \multicolumn{3}{|c|}{$\begin{array}{l}\text { Micronutrient } \\
\text { (ppm) }\end{array}$} & \multicolumn{3}{|c|}{$\begin{array}{c}\text { Macro } \\
\text { nutrient } \\
\text { (ppm) }\end{array}$} & \multicolumn{4}{|c|}{ Soluble anions(meq/l) } & \multicolumn{4}{|c|}{$\begin{array}{c}\text { Soluble } \\
\text { Cations (meq/l) }\end{array}$} & \multirow[t]{2}{*}{$\begin{array}{c}\mathrm{Caco3} \\
\%\end{array}$} & \multirow{2}{*}{$\begin{array}{c}\text { E.C } \\
\text { ds/ } \\
\text { m }\end{array}$} & \multirow[t]{2}{*}{$\begin{array}{l}\mathbf{P} \\
\mathbf{H}\end{array}$} \\
\hline Mn & $\mathbf{Z n}$ & $\mathbf{F e}$ & $\mathbf{N}$ & $\mathbf{P}$ & $\mathbf{K}$ & $\mathrm{So4}^{-}$ & Cl & Hco3 & $\begin{array}{c}\mathrm{Co3} \\
-.\end{array}$ & $\mathbf{K}^{+}$ & $\begin{array}{c}\mathbf{N a} \\
++\end{array}$ & $\underset{++}{\mathrm{Mg}}$ & $\begin{array}{c}\mathbf{C a}^{+} \\
+\end{array}$ & & & \\
\hline 2.55 & 1.2 & 6.4 & 100 & 50 & 448 & 1.64 & 5.7 & 1.4 & - & 0.49 & 2.1 & 0.2 & 1 & 7.8 & 0.32 & 7.5 \\
\hline
\end{tabular}

Table 2. chemical analysis of compost used at experimental period.

\begin{tabular}{cccccc}
\hline \multicolumn{2}{c}{ (Micronutrient ) } & Ppm & \multicolumn{3}{c}{ Macro nutrient \% } \\
\hline Zn & Mn & Fe & K & P & N \\
\hline 26 & 5.2 & 1938 & 1.36 & 0.14 & 1.26 \\
\hline O.C \% & \multicolumn{3}{c}{ O.M \% } & & C/N ratio \\
\hline 13.2 & & 22.8 & 10.5 \\
\hline
\end{tabular}

Plant material: seeds of orange flesh melon (Magenta. $F_{1}$ hybrid ) were obtained through Nunhems Co. and sown in the nursery using foam seedling trays (84 cells) which were filled with a mixture of peat moss: vermiculite $(1: 1 \mathrm{~V} / \mathrm{V})$ on May $7^{\text {th }}$ and May ${ }^{\text {st }}$ in 2011 and 2012, respectively. The seedlings were transplanted in the field on May $29^{\text {th }} 2011$ and May $23^{\text {rd }} 2012$ on the centre of the bed. The area of each plot was $12 \mathrm{~m}^{2}(10 \mathrm{~m}$ long $\times 1.20 \mathrm{~m}$ width). Each plot contained 20 plants spacing of $50 \mathrm{~cm}$ between hills within the row as plant. All missing plants were replaced by seedling. Drip irrigation method and black plastic mulch were used.

The experiments included 24 treatments arranged in split-split plot design (using three replicates for each treatment) as follows:

Main plots treatment were filled with 6 different organic (compost) and inorganic $(\mathrm{N})$ rates/ fed. as follows:

1. 5 ton compost +zero Nitrogen/fed

2. 4 ton compost $+20 \mathrm{~kg} \mathrm{Nitrogen} / \mathrm{fed}$

3. 3 ton compost $+40 \mathrm{~kg}$ Nitrogen $/$ fed

4. 2 ton compost $+60 \mathrm{~kg} \mathrm{Nitrogen} / \mathrm{fed}$

5. 1 ton compost $+80 \mathrm{~kg}$ Nitrogen/fed

6. zero ton compost $+100 \mathrm{~kg}$ Nitrogen $/ \mathrm{fed}$

2- sub main plot treatments were filled with two rates of potassium (salphate potassium) as follows:

1. $100 \mathrm{~kg} \mathrm{~K}_{2} \mathrm{O} /$ fed

2. $150 \mathrm{~kg} \mathrm{~K} 2 \mathrm{O} /$ fed

3- Sub-sub main plot treatments were included two rates of calcium ( Edeta )foliar application as follows:

1-water spraying (expressed as zero calcium)

2- Calcium spraying with the rate of 400 $\mathrm{g} / 100$ liter/fed
$60 \mathrm{Kg} / \mathrm{fed}$.phosphorus were added to all plots among experimental units

The compost and phosphor were applied during soil preparation. The quantity of mineral $\mathrm{N}$ and $\mathrm{K}$ fertilizer in each treatment was divided into two equal doses 21 and added at 45 days after sowing. The treatment of foliar calcium was applied three times (30, 40, 50 days after sowing)

\section{Data recorded}

1-vegetative growth parameters.

Three plants were randomly taken from each plot for measuring the following vegetative growth parameters after 60 days from transplanting

- Plant length

- Number of leaves per plant

- Plant fresh weight

- Plant dry weight

- Yield and its components

Harvesting date began after 60 days from transplanting and yield and its component were recorded expressed as:

- Number of fruits/plant

- Average fruit weight

- Yield/plant and yield/fed

\section{Result and discussion}

\section{Vegetative growth parameters}

\section{Effect of organic and mineral fertilization}

1- Plant length: Data presented in Table 3 indicate that the effect of organic and mineral fertilization on plant length was significant in 
both growing seasons. In this respect ,the application of 2 ton compost $+60 \mathrm{~kg} \mathrm{~N}$ gave the highest plant length in both seasons. In addition the same trend was recorded with using 3 ton compost $+40 \mathrm{~kg} \mathrm{~N} /$ fed in the first season.

2- Number of leaves per plant : Data presented in Table 3 indicate that melon plant that fertilized with 2 ton compost $+60 \mathrm{~kg} \mathrm{~N}$ gave the highest number of leaves in both seasons

3- Plant fresh weight: Data in the same Table indicate that melon plants that received 2 ton compost $+60 \mathrm{~kg} \mathrm{~N}$ were the heaviest in both seasons

4- Plant dry weight: Data in Table 3 indicate that the application of 2 ton compost $+60 \mathrm{~kg} \mathrm{~N}$ gave the highest plant dry weight in both seasons.

Generally the lowest values of plant length, number of leaves, plant fresh and dry weight were recorded with 1 ton compost + zero $\mathrm{N}$ in both seasons

Table 3. Effect of organic and mineral, potassium and calcium foliar application on vegetative growth parameters.

\begin{tabular}{|c|c|c|c|c|c|c|c|c|c|}
\hline \multirow{3}{*}{ Treatments } & \multicolumn{9}{|c|}{ Vegetative growth } \\
\hline & \multicolumn{3}{|c|}{ Plant length(m) } & \multicolumn{2}{|c|}{$\begin{array}{l}\text { Number of } \\
\text { leaves/plant }\end{array}$} & \multicolumn{2}{|c|}{$\begin{array}{c}\text { Plant fresh } \\
\text { weight (g) }\end{array}$} & \multicolumn{2}{|c|}{$\begin{array}{c}\text { Plant dry } \\
\text { weight(g) }\end{array}$} \\
\hline & & $\begin{array}{c}\text { Season } \\
2011\end{array}$ & $\begin{array}{c}\text { Season } \\
2012\end{array}$ & $\begin{array}{c}\text { Season } \\
2011\end{array}$ & $\begin{array}{c}\text { Season } \\
2012\end{array}$ & $\begin{array}{c}\text { Season } \\
2011\end{array}$ & $\begin{array}{c}\text { Season } \\
2012\end{array}$ & $\begin{array}{c}\text { Season } \\
2011\end{array}$ & $\begin{array}{c}\text { Season } \\
2012\end{array}$ \\
\hline \multirow{6}{*}{$\begin{array}{l}\text { Compost and } \\
\mathrm{N} \text { rates }\end{array}$} & 1 & $1.59 \mathrm{c}$ & $1.81 \mathrm{~d}$ & $190.8 \mathrm{~d}$ & $201.6 \mathrm{~d}$ & $1402 \mathrm{~d}$ & $1415 \mathrm{~d}$ & $336.2 \mathrm{~d}$ & $362.8 \mathrm{~cd}$ \\
\hline & 2 & $1.75 \mathrm{bc}$ & $1.96 \mathrm{c}$ & $214.8 \mathrm{c}$ & $222.8 \mathrm{c}$ & $1457 \mathrm{bc}$ & $1481 \mathrm{~b}$ & $367.6 \mathrm{~b}$ & $370.2 \mathrm{bc}$ \\
\hline & 3 & $1.98 \mathrm{ab}$ & $2.21 \mathrm{~b}$ & $229.3 \mathrm{~b}$ & $240.7 \mathrm{~b}$ & $1471 \mathrm{~b}$ & $1496 \mathrm{~b}$ & $372.4 \mathrm{~b}$ & $379.0 \mathrm{~b}$ \\
\hline & 4 & $2.22 \mathrm{a}$ & $2.45 \mathrm{a}$ & $253.3 \mathrm{a}$ & $262.3 \mathrm{a}$ & $1543 \mathrm{a}$ & $1541 \mathrm{a}$ & $391.5 \mathrm{a}$ & $400.5 \mathrm{a}$ \\
\hline & 5 & $1.86 \mathrm{~b}$ & $2.13 \mathrm{~b}$ & $230.8 \mathrm{~b}$ & $241.3 \mathrm{~b}$ & $1466 \mathrm{~b}$ & $1474 \mathrm{~b}$ & $368.4 \mathrm{~b}$ & $368.2 \mathrm{c}$ \\
\hline & 6 & $1.89 \mathrm{~b}$ & $1.97 \mathrm{c}$ & $213.4 \mathrm{c}$ & $235.0 \mathrm{~b}$ & $1432 \mathrm{~cd}$ & $1442 \mathrm{c}$ & $360.3 \mathrm{c}$ & $358.4 \mathrm{~d}$ \\
\hline L.S.D(0.05) & & 0.24 & 0.10 & 10.70 & 7.50 & 31.0 & 26.5 & 6.83 & 9.019 \\
\hline \multirow{2}{*}{$\begin{array}{l}\text { Potassium } \\
\text { Levels kg/fed }\end{array}$} & 100 & $1.83 \mathrm{~b}$ & $2.05 \mathrm{~b}$ & $217.1 \mathrm{~b}$ & $229.0 \mathrm{~b}$ & $1440.0 \mathrm{~b}$ & $1460 \mathrm{~b}$ & $362.0 \mathrm{~b}$ & $368.3 \mathrm{~b}$ \\
\hline & 150 & $1.93 \mathrm{a}$ & $2.13 \mathrm{a}$ & $227 \mathrm{a}$ & $238.9 \mathrm{a}$ & $1483.6 \mathrm{a}$ & $1490 \mathrm{a}$ & $370.1 \mathrm{a}$ & $378.00 \mathrm{a}$ \\
\hline L.S.D(0.05) & & 0.09 & 0.06 & 5.9 & 2.9 & 13.08 & 12.63 & 4.32 & 9.408 \\
\hline \multirow{2}{*}{$\begin{array}{l}\text { Calcium } \\
\text { levels }(400 \mathrm{~g} / \mathrm{fed})\end{array}$} & unsprayd & $1.86 \mathrm{a}$ & $2.06 \mathrm{a}$ & $2.20 \mathrm{a}$ & $231.6 \mathrm{a}$ & $1448.2 \mathrm{~b}$ & $1460 \mathrm{~b}$ & $363.4 \mathrm{~b}$ & $368.3 \mathrm{~b}$ \\
\hline & sprayed & $1.90 \mathrm{a}$ & $2.12 \mathrm{a}$ & $224.1 \mathrm{a}$ & $236.3 \mathrm{a}$ & $1475.4 \mathrm{a}$ & $1490 \mathrm{a}$ & $368.7 \mathrm{a}$ & $378.00 \mathrm{a}$ \\
\hline L.S.D(0.05) & & 0.09 & $\mathbf{0 . 0 7}$ & 4.75 & 6.0 & 14.88 & 12.91 & 4.164 & 7.091 \\
\hline $\begin{array}{l}\text { Compost and } \mathrm{N} \mathrm{ra} \\
1=(5 \text { ton compost } \\
4=(2 \text { ton compost }\end{array}$ & $\begin{array}{l}\text { zero N) /fed } \\
60 \mathrm{~kg} \mathrm{~N} \text { )/fed }\end{array}$ & & (1 ton com & $\begin{array}{l}\text { ost }+20 \mathrm{k} \\
\text { post }+80 \mathrm{k}\end{array}$ & $\begin{array}{l}\text { N)/fed } \\
\text { N) /fed }\end{array}$ & $\begin{array}{r}3=(3 \\
6=(\end{array}$ & $\begin{array}{l}\text { ton comp } \\
\text { ero ton co }\end{array}$ & $\begin{array}{l}\mathrm{t}+40 \mathrm{~kg} \mathrm{I} \\
\text { upost }+10\end{array}$ & $\begin{array}{l}\text { /fed } \\
\mathrm{kg} \mathrm{N} \text { )/fed }\end{array}$ \\
\hline
\end{tabular}

Vegetative growths parameters values were higher with the application of compost, Roe and Comforth (1997) Mohamed (2012) on cantaloupe and Bayoumi and Hafez( 2006), Jahromi et al .(2012)on cucumber. On the other hand Abd El-Aziz (2003) found that organic manure decreased cantaloupe plant height and number of leaves compared to inorganic solution in both seasons.

These results may be due to the favorable effect of organic manure on soil physical and chemical properties. The structural improvement can encourage the plant to have a good root development, which lead to a higher plant vegetative growth .similar results were obtained by EL-Shafie and ELGammaily (2002) on onion, Hafez and Mahmoud( 2003) on squash plants .The same results were obtained by Abdallah et al .(2006) on cucumber plants Abo EL-Nour (2008 )on cantaloupe found similar results.

Tindall (1968) stated that relatively high levels of nutrients are required for optimum growth and development at early stage. With compost, nutrient element content is low and the nutrients are not readily available for plant uptakes (Brady, 1990)

Organic manures activates many species of living organisms which release phytohormones and may stimulate the plant growth and absorption of nutrients ( Arisha et al., 2003)

The combination of organic materials with reduced NPK fertilizer rates produced plants that were similar to unmixed NPK fertilizer. This indicated that the high dose of organic manures can be reduced by half and mixed with a reduced rate of NPK fertilizers as reported by Akande et al. (2003).

Generally improving vegetative growth parameters by treatment of compost plus chemical $\mathrm{N}$ fertilizer compared with using each alone can be attributed to that applying mineral $\mathrm{N}$ stimulated the rate of decomposition of compost and produced higher humus substances which improve the physical and chemical properties of the soil as well as increase both the exchangeable water soluble of nutrients and their uptake (Cooke, 1972). In this concern, Nafadi and Gohar (1975) reported that adsorption $\mathrm{NH}^{+}$ion on the surface of compost and became available to plant uptake.

Effect of potassium level .

As for the effect of potassium levels on vegetative growth character, data in Table 3 show 
that the highest plant lengths, number of leaves/plant, plant fresh and dry weight were recorded with $150 \mathrm{~kg}$ $\mathrm{K}_{2} \mathrm{O}$ in both seasons

\section{Effect of calcium foliar application.}

Data presented in Table 3 indicate that foliar of calcium treatment had no significant effect on plant length and number of leaves in both seasons, but the highest plant fresh and dry weight were recorded with foliar calcium treatment of $400 \mathrm{~g} / \mathrm{fed}$ in both seasons.
Effect of the interaction between organic and mineral fertilization and potassium levels

The effect of the interaction between organic and mineral and potassium level on vegetative growth were significant in both growing seasons. Table 4 and 5 show that the highest values of plant length, number of leaves, plant fresh and dry weight were recorded with 2 ton compost $+60 \mathrm{~kg} \mathrm{~N}$ and fertilized with $150 \mathrm{~kg} / \mathrm{fed}$ of potassium in both seasons while the lowest values of these vegetative growth characters were recorded with 5 ton compost + zero $\mathrm{N}$ and fertilized by $100 \mathrm{~kg} \mathrm{~K}_{2} \mathrm{O} /$ fed in both seasons

Table 4. Effect of interaction between organic and mineral fertilizer and potassium level on plant length and number of leaves.

\begin{tabular}{|c|c|c|c|c|c|c|c|c|}
\hline \multirow{4}{*}{$\begin{array}{c}\text { Compost and } \\
\text { N rates }\end{array}$} & \multicolumn{4}{|c|}{ Plant length(m) } & \multicolumn{4}{|c|}{ Number of leaves/plant } \\
\hline & \multicolumn{2}{|c|}{2011} & \multicolumn{2}{|c|}{2012} & \multicolumn{2}{|c|}{2011} & \multicolumn{2}{|c|}{2012} \\
\hline & \multicolumn{8}{|c|}{ Potassium levels (kg/fed) } \\
\hline & 100 & 150 & 100 & 150 & 100 & 150 & 100 & 150 \\
\hline 1 & $1.52 \mathrm{f}$ & $1.66 \mathrm{ef}$ & $1.73 \mathrm{f}$ & 1.89 ef & $185.3 \mathrm{~h}$ & $196.3 \mathrm{gh}$ & $193.7 \mathrm{j}$ & $209.5 \mathrm{i}$ \\
\hline 2 & $1.73 \mathrm{def}$ & $1.78 \mathrm{de}$ & $1.97 \mathrm{de}$ & $1.94 \mathrm{e}$ & $210.5 \mathrm{efg}$ & $219.0 \mathrm{def}$ & $218.3 \mathrm{~h}$ & $227.2 \mathrm{~g}$ \\
\hline 3 & $1.92 \mathrm{bcd}$ & $2.04 \mathrm{bc}$ & $2.13 \mathrm{~d}$ & $2.29 \mathrm{bc}$ & $221.7 \mathrm{de}$ & $237.0 \mathrm{bc}$ & $231.5 \mathrm{fg}$ & $249.8 \mathrm{c}$ \\
\hline 4 & $2.13 \mathrm{ab}$ & $2.30 \mathrm{a}$ & $2.37 \mathrm{~b}$ & $2.53 \mathrm{a}$ & $248.8 \mathrm{ab}$ & $257.7 \mathrm{a}$ & $258.2 \mathrm{~b}$ & $266.5 \mathrm{a}$ \\
\hline 5 & $1.84 \mathrm{cde}$ & $1.89 \mathrm{cde}$ & $2.14 \mathrm{c}$ & $2.13 \mathrm{~d}$ & $229.7 \mathrm{~cd}$ & $232.0 \mathrm{~cd}$ & $239.0 \mathrm{de}$ & $243.7 \mathrm{~cd}$ \\
\hline 6 & $1.87 \mathrm{cde}$ & $1.91 \mathrm{bcd}$ & $1.94 \mathrm{e}$ & $2.00 \mathrm{de}$ & $206.8 \mathrm{fg}$ & $220.0 \mathrm{def}$ & $233.5 \mathrm{efg}$ & $236.5 \mathrm{ef}$ \\
\hline
\end{tabular}

L.S.D(0.05) 0.22 0.16 14.53 7.12

Compost and $\mathrm{N}$ rates:

$1=(5$ ton compost + zero $\mathrm{N}) /$ fed

$4=(2$ ton compost $+60 \mathrm{~kg} \mathrm{~N}) / \mathrm{fed}$
$2=(4$ ton compost $+20 \mathrm{~kg} \mathrm{~N}) / \mathrm{fed}$

$5=(1$ ton compost $+80 \mathrm{~kg} \mathrm{~N}) /$ fed
$3=(3$ ton compost $+40 \mathrm{~kg} \mathrm{~N}) /$ fed

$6=($ zero ton compost $+100 \mathrm{~kg} \mathrm{~N}) /$ fed

Table 5. Effect of interaction between organic and mineral fertilizer and potassium level on plant fresh and dry weight.

\begin{tabular}{|c|c|c|c|c|c|c|c|c|}
\hline \multirow{4}{*}{$\begin{array}{c}\text { Compost and } \\
\mathrm{N} \text { rates }\end{array}$} & \multicolumn{4}{|c|}{ Plant fresh weight $(g)$} & \multicolumn{4}{|c|}{ Plant dry weight(g) } \\
\hline & \multicolumn{2}{|c|}{2011} & \multicolumn{2}{|c|}{2012} & \multicolumn{2}{|c|}{2011} & \multicolumn{2}{|c|}{2012} \\
\hline & \multicolumn{8}{|c|}{ Potassium levels( kg/fed) } \\
\hline & 100 & 150 & 100 & 150 & 100 & 150 & 100 & 150 \\
\hline 1 & $1361 \mathrm{~h}$ & 1443 efg & $1400 \mathrm{f}$ & 1430 ef & $330.3 \mathrm{f}$ & $342.1 \mathrm{e}$ & $357 \mathrm{e}$ & $368.6 \mathrm{cde}$ \\
\hline 2 & $1422 \mathrm{~g}$ & $1492 \mathrm{bc}$ & $1469 \mathrm{~cd}$ & $1492 \mathrm{bc}$ & $365.8 \mathrm{bcd}$ & $369.3 \mathrm{bc}$ & $365.7 \mathrm{de}$ & $374.7 \mathrm{~cd}$ \\
\hline 3 & 1457 def & 1484bcd & $1478 \mathrm{c}$ & $1513 \mathrm{~b}$ & $370.3 \mathrm{bc}$ & $374.5 \mathrm{~b}$ & $374 \mathrm{cde}$ & $384.1 \mathrm{bc}$ \\
\hline 4 & $1515 \mathrm{~b}$ & $1572 \mathrm{a}$ & $1484 \mathrm{bc}$ & $1599 \mathrm{a}$ & $391.8 \mathrm{a}$ & $391.1 \mathrm{a}$ & $397.1 \mathrm{ab}$ & $403.8 \mathrm{a}$ \\
\hline 5 & $1458 \mathrm{def}$ & $1475 \mathrm{cde}$ & $1482 \mathrm{c}$ & $1466 \mathrm{~cd}$ & $364.5 \mathrm{bcd}$ & $372.3 \mathrm{bc}$ & $359.6 \mathrm{de}$ & $376.8 \mathrm{~cd}$ \\
\hline 6 & $1428 \mathrm{fg}$ & $1436 \mathrm{fg}$ & $1444 \mathrm{de}$ & $1441 \mathrm{de}$ & $357.7 \mathrm{~d}$ & $363.0 \mathrm{~cd}$ & $356.8 \mathrm{e}$ & $360.1 \mathrm{de}$ \\
\hline L.S.D(0.05) & \multicolumn{2}{|c|}{32.05} & \multicolumn{2}{|c|}{30.94} & \multicolumn{2}{|c|}{10.20} & \multicolumn{2}{|c|}{17.37} \\
\hline $\begin{array}{l}\text { Compost and } N \text { rate } \\
1=(5 \text { ton compost }+ \\
4=(2 \text { ton compost }\end{array}$ & $\begin{array}{l}\text { ro N)/fed } \\
(0 \mathrm{~kg} \mathrm{~N}) / \text { fed }\end{array}$ & $\begin{array}{l}2=(4 \text { to } \\
5=(1 \text { to }\end{array}$ & $\begin{array}{l}\text { ompost }+20 \mathrm{k} \\
\text { ompost }+80 \mathrm{l}\end{array}$ & $\begin{array}{l}\text { N)/fed } \\
\text { N) /fed }\end{array}$ & $\begin{array}{l}3=(3 \text { ton } \\
6=(\text { zero }\end{array}$ & $\begin{array}{l}\text { ompost }+40 \\
\text { n compost }+\end{array}$ & $\begin{array}{l}\text { N) /fed } \\
00 \mathrm{~kg} \mathrm{~N}) / \mathrm{fed}\end{array}$ & \\
\hline
\end{tabular}

\section{Effect of the interaction between organic and mineral and calcium foliar application on vegetative growth parameters}

Data in Table 6 show the effect of the interaction between organic and mineral fertilizer and calcium foliar application on vegetative growth characters. Significant differences in both seasons were recorded on these parameters(plant length, number of leaves/plant , plant fresh and dry weight) with 2 ton compost $+60 \mathrm{~kg} \mathrm{~N}$ with calcium treatments $(0$ and $400 \mathrm{~g} / \mathrm{fed}$ ) in season of 2011 and 2012. Also the treatment of 3 ton compost $+40 \mathrm{~kg} \mathrm{~N}$ and sprayed with calcium at $400 \mathrm{~g} /$ fed produced the highest plant length in the first season, but the lowest values of these characters were recorded with unsprayed plants with calcium and treated with 5 ton compost + zero $\mathrm{N}$ 
Table 6 .Effect of interaction between organic and mineral and calcium foliar application on plant length and number of leaves .

\begin{tabular}{|c|c|c|c|c|c|c|c|c|}
\hline \multirow{4}{*}{$\begin{array}{c}\text { Compost } \\
\text { and } \\
\mathbf{N} \text { rates }\end{array}$} & \multicolumn{4}{|c|}{ Plant length(m) } & \multicolumn{4}{|c|}{ Number of leaves/plant } \\
\hline & \multicolumn{2}{|c|}{2011} & \multicolumn{2}{|c|}{2012} & \multicolumn{2}{|c|}{2011} & \multicolumn{2}{|c|}{2012} \\
\hline & \multicolumn{8}{|c|}{ Calcium levels(400g/fed) } \\
\hline & $\begin{array}{c}\text { unspraye } \\
\text { d }\end{array}$ & $\begin{array}{c}\text { spraye } \\
\text { d }\end{array}$ & $\begin{array}{c}\text { unspraye } \\
\text { d }\end{array}$ & $\begin{array}{c}\text { spraye } \\
\text { d }\end{array}$ & $\begin{array}{c}\text { unspraye } \\
\text { d }\end{array}$ & sprayed & $\begin{array}{c}\text { unspray } \\
\text { ed }\end{array}$ & $\begin{array}{c}\text { Spraye } \\
\text { d }\end{array}$ \\
\hline 1 & $1.58 \mathrm{e}$ & $1.60 \mathrm{de}$ & $1.75 \mathrm{~g}$ & $1.87 \mathrm{fg}$ & $189.8 \mathrm{f}$ & $191.8 \mathrm{f}$ & $200.5 \mathrm{f}$ & $202.7 \mathrm{f}$ \\
\hline 2 & $1.72 \mathrm{cde}$ & $1.79 \mathrm{~b}-\mathrm{e}$ & $1.92 \mathrm{fg}$ & $1.99 \mathrm{def}$ & $214.7 \mathrm{de}$ & 214.c de & $218.3 \mathrm{e}$ & $227.3 \mathrm{de}$ \\
\hline 3 & $1.95 \mathrm{bc}$ & $2.01 \mathrm{ab}$ & $2.19 \mathrm{c}$ & $2.23 \mathrm{bc}$ & $226.7 \mathrm{bc}$ & $232 \mathrm{~b}$ & $235.2 \mathrm{~cd}$ & $246.2 \mathrm{bc}$ \\
\hline 4 & $2.20 \mathrm{a}$ & $2.24 \mathrm{a}$ & $2.39 \mathrm{ab}$ & $2.51 \mathrm{a}$ & $251.2 \mathrm{a}$ & $255.3 \mathrm{a}$ & $260.5 \mathrm{ab}$ & $264.2 \mathrm{a}$ \\
\hline 5 & $1.83 \mathrm{bcd}$ & $1.90 \mathrm{bc}$ & $2.12 \mathrm{cde}$ & $2.15 \mathrm{~cd}$ & $232.8 \mathrm{~b}$ & $228.8 \mathrm{~b}$ & $241.5 \mathrm{~cd}$ & $241.2 \mathrm{~cd}$ \\
\hline 6 & $1.91 \mathrm{bc}$ & $1.87 \mathrm{bc}$ & $1.96 \mathrm{ef}$ & $1.99 \mathrm{def}$ & 205.5 & $221.3 \mathrm{bcd}$ & $234 \mathrm{~cd}$ & $234.3 \mathrm{~cd}$ \\
\hline L.S.D(0.05) & \multicolumn{2}{|c|}{0.23} & \multicolumn{2}{|c|}{0.18} & \multicolumn{2}{|c|}{11.57} & \multicolumn{2}{|c|}{14.79} \\
\hline \multicolumn{3}{|c|}{$\begin{array}{l}\text { Compost and } N \text { rates: } \\
1=(5 \text { ton compost }+ \text { zero } N) / \text { fed } \\
4=(2 \text { ton compost }+60 \mathrm{~kg} \mathrm{~N}) / \mathrm{fed}\end{array}$} & $\begin{array}{l}2=(4 \text { ton } \mathrm{co} \\
5=(1 \text { ton } \mathrm{co}\end{array}$ & $\begin{array}{l}\text { post }+20 \mathrm{k} \\
\text { ost }+80 \mathrm{~kg}\end{array}$ & $\begin{array}{l}\text { N)/fed } \\
\text { J) /fed }\end{array}$ & \multicolumn{3}{|c|}{$\begin{array}{c}3=(3 \text { ton compost }+40 \mathrm{~kg} \mathrm{~N}) / \mathrm{fed} \\
6=(\text { zero ton compost }+100 \mathrm{~kg} \mathrm{~N}) / \mathrm{fed}\end{array}$} \\
\hline
\end{tabular}

Table 7. Effect of interaction between organic and mineral and calcium foliar application on plant fresh and dry weight.

\begin{tabular}{|c|c|c|c|c|c|c|c|c|}
\hline \multirow{4}{*}{$\begin{array}{c}\text { Compost } \\
\text { and } \\
\mathbf{N} \text { rates }\end{array}$} & \multicolumn{4}{|c|}{ Plant fresh weigh(g) } & \multicolumn{4}{|c|}{ Plant dry weight(g) } \\
\hline & \multicolumn{2}{|c|}{2011} & \multicolumn{2}{|c|}{2012} & \multicolumn{2}{|c|}{2011} & \multicolumn{2}{|c|}{2012} \\
\hline & \multicolumn{8}{|c|}{ Calcium levels $(400 \mathrm{~g} / \mathrm{fed})$} \\
\hline & $\begin{array}{c}\text { unspraye } \\
\text { d }\end{array}$ & sprayed & $\begin{array}{c}\text { unspraye } \\
\text { d }\end{array}$ & $\begin{array}{c}\text { spraye } \\
\text { d }\end{array}$ & $\begin{array}{c}\text { unspraye } \\
\text { d }\end{array}$ & $\begin{array}{c}\text { spraye } \\
\text { d }\end{array}$ & $\begin{array}{c}\text { unspraye } \\
\text { d }\end{array}$ & Sprayed \\
\hline 1 & $1373 \mathrm{f}$ & $1430 \mathrm{de}$ & $1406 \mathrm{~g}$ & $1425 \mathrm{fg}$ & $330.3 \mathrm{f}$ & $342.1 \mathrm{e}$ & $357 \mathrm{e}$ & $\begin{array}{l}368.6 \mathrm{~cd} \\
\mathrm{e}\end{array}$ \\
\hline 2 & 1444 cde & $1470 \mathrm{bc}$ & $1462 \mathrm{de}$ & 1499 bc & $365.8 \mathrm{bcd}$ & $369.3 \mathrm{bc}$ & $365.7 \mathrm{de}$ & $374.7 \mathrm{~cd}$ \\
\hline 3 & 1452 cde & $1489 \mathrm{~b}$ & $1480 \mathrm{~cd}$ & $1512 \mathrm{~b}$ & $370.3 \mathrm{bc}$ & $374.5 \mathrm{~b}$ & 374 cde & $384.1 \mathrm{bc}$ \\
\hline 4 & $1544 \mathrm{a}$ & $1543 \mathrm{a}$ & $1527 \mathrm{ab}$ & $1556 \mathrm{a}$ & $391.8 \mathrm{a}$ & $391.1 \mathrm{a}$ & $397.1 \mathrm{ab}$ & $403.8 \mathrm{a}$ \\
\hline 5 & 1458 bcd & $1475 \mathrm{bc}$ & 1438 ef & $1510 \mathrm{bc}$ & $364.5 \mathrm{bcd}$ & $372.3 \mathrm{bc}$ & $359.6 \mathrm{de}$ & $376.8 \mathrm{~cd}$ \\
\hline 6 & $1419 \mathrm{e}$ & $1446 \mathrm{cde}$ & 1447 ef & $1438 \mathrm{f}$ & $357.7 \mathrm{~d}$ & $363.0 \mathrm{~cd}$ & $356.8 \mathrm{e}$ & $360.1 \mathrm{de}$ \\
\hline L.S.D(0.05) & \multicolumn{2}{|c|}{36.46} & \multicolumn{2}{|c|}{31.62} & \multicolumn{2}{|c|}{10.20} & \multicolumn{2}{|c|}{17.37} \\
\hline \multicolumn{3}{|c|}{$\begin{array}{l}\text { Compost and N rates: } \\
1=(5 \text { ton compost }+ \text { zero } N) / \text { fed } \\
4=(2 \text { ton compost }+60 \mathrm{~kg} \mathrm{~N}) / \mathrm{fed}\end{array}$} & $\begin{array}{r}2=(4 \text { ton com } \\
5=(1 \text { ton comp }\end{array}$ & $\begin{array}{l}\text { ost }+20 \mathrm{~kg} \\
\mathrm{st}+80 \mathrm{~kg} \mathrm{I}\end{array}$ & \multicolumn{4}{|c|}{$\begin{array}{l}3=(3 \text { ton compost }+40 \mathrm{~kg} \mathrm{~N}) / \mathrm{fed} \\
6=(\text { zero ton compost }+100 \mathrm{~kg} \mathrm{~N}) / \mathrm{fed}\end{array}$} \\
\hline
\end{tabular}

\section{Effect of the interaction between potassium and calcium foliar application}

With respect to the effect of the interaction between potassium and calcium foliar application, data in Table 8 and 9 indicate that there were no significant differences on plant length due to calcium treatments and potassium rates. The highest numbers of leaves were recorded with $150 \mathrm{~kg} \mathrm{~K}_{2} \mathrm{O}$ with unsprayed and sprayed plants in both seasons. Moreover the highest values of fresh weight were recorded when plants fertilized with $150 \mathrm{~kg} \mathrm{~K}_{2} \mathrm{O}$ and sprayed with400 g calcium/fed in both season. Meanwhile the highest values of dry weight were recorded when plants received $150 \mathrm{~kg} \quad \mathrm{~K}_{2} \mathrm{O}$ and sprayed with $400 \mathrm{~g}$ calcium/fed and untreated plants in the first season. But in the second season there were non significant differences between $100 \mathrm{~kg} \mathrm{~K}_{2} \mathrm{O}$ with $400 \mathrm{~g}$ calcium/fed and $150 \mathrm{~kg} \mathrm{~K} \mathrm{~K}_{2} \mathrm{O}$ with sprayed and unsprayed with calcium. The lowest values of fresh and dry weight were recorded when plants received $100 \mathrm{~kg} \mathrm{~K}_{2} \mathrm{O}$ and non treated plants with calcium $400 \mathrm{~g} / \mathrm{fed}$ in both seasons.

Table 8. Effect of the interaction between potassium and calcium foliar application plant length and number of leaves.

\begin{tabular}{|c|c|c|c|c|c|c|c|c|}
\hline \multirow{4}{*}{$\begin{array}{l}\text { Potassium } \\
\text { Level } \\
\text { (kg/fed) }\end{array}$} & \multicolumn{4}{|c|}{ Plant length(m) } & \multicolumn{4}{|c|}{ Number of leaves/plant } \\
\hline & \multicolumn{2}{|c|}{2011} & \multicolumn{2}{|c|}{2012} & \multicolumn{2}{|c|}{2011} & \multicolumn{2}{|c|}{2012} \\
\hline & \multicolumn{8}{|c|}{$\begin{array}{c}\text { Calcium level }(400 \mathrm{~g} / \mathrm{fed}) \\
\end{array}$} \\
\hline & unsprayed & sprayed & unsprayed & sprayed & unsprayed & sprayed & unsprayed & Sprayed \\
\hline 100 & $1.83 \mathrm{a}$ & $1.84 \mathrm{a}$ & $2.02 \mathrm{~b}$ & $2.07 \mathrm{ab}$ & $212.9 \mathrm{~b}$ & $221.9 \mathrm{~b}$ & $226.4 \mathrm{c}$ & $231.7 \mathrm{bc}$ \\
\hline 150 & $1.90 \mathrm{a}$ & $1.96 \mathrm{a}$ & $2.09 \mathrm{ab}$ & $2.17 \mathrm{a}$ & $225.4 \mathrm{a}$ & $228.8 \mathrm{a}$ & $236.9 \mathrm{ab}$ & $240.8 \mathrm{a}$ \\
\hline L.S.D(0.05) & \multicolumn{2}{|c|}{ N.S. } & \multicolumn{2}{|c|}{ N.S. } & \multicolumn{2}{|c|}{6.6} & \multicolumn{2}{|c|}{8.5} \\
\hline
\end{tabular}


Table (9) Effect of the interaction between potassium and calcium foliar application on plant fresh and dry weight.

\begin{tabular}{|c|c|c|c|c|c|c|c|c|}
\hline \multirow{4}{*}{$\begin{array}{l}\text { Potassium } \\
\text { Level } \\
\text { (kg/fed) }\end{array}$} & \multicolumn{4}{|c|}{ Plant fresh weight $(g)$} & \multicolumn{4}{|c|}{ Plant dry weight(g) } \\
\hline & \multicolumn{2}{|c|}{2011} & \multicolumn{2}{|c|}{2012} & \multicolumn{2}{|c|}{2011} & \multicolumn{2}{|c|}{2012} \\
\hline & \multicolumn{8}{|c|}{$\begin{array}{c}\text { Calcium level }(400 \mathrm{~g} / \mathrm{fed}) \\
\end{array}$} \\
\hline & unsprayed & sprayed & unsprayed & sprayed & unsprayed & sprayed & unsprayed & sprayed \\
\hline 100 & $1425 \mathrm{c}$ & $1455 \mathrm{~b}$ & $1442 \mathrm{c}$ & $1477 \mathrm{~b}$ & $358.4 \mathrm{~b}$ & 365.6 & $362.8 \mathrm{~b}$ & $373.9 \mathrm{a}$ \\
\hline 150 & $1471 \mathrm{~b}$ & $1496 \mathrm{a}$ & $1478 \mathrm{~b}$ & $1502 \mathrm{a}$ & 367.7 & $372.0 \mathrm{a}$ & $373.9 \mathrm{a}$ & $382.1 \mathrm{a}$ \\
\hline L.S.D(0.05) & \multicolumn{2}{|c|}{21.05} & \multicolumn{2}{|c|}{18.25} & \multicolumn{2}{|c|}{11.01} & \multicolumn{2}{|c|}{10.03} \\
\hline
\end{tabular}

Effect of the interaction between organic and nitrogen, potassium and calcium

With respect to the effect of the interaction between organic and nitrogen, potassium and calcium foliar application, data in Table 10 and 11 indicate that the highest plant length, number of leaves, plant fresh and dry weight were recorded with 2 ton compost $+60 \mathrm{~kg} \mathrm{~N}$ and fertilized by both potassium levels and sprayed with calcium in both seasons.
Effect of organic -mineral fertilization, potassium level, calcium foliar application on number of fruits and average fruit weight

Number of fruits and average fruit weight

It appears from data in Table 12 that fertilizing plants with 2 ton compost $+60 \mathrm{~kg} \mathrm{~N}$ and 3 ton compost $+40 \mathrm{~kg} \mathrm{~N}$ gave the highest number of fruits and average fruit weight in the both seasons. and also 3 ton compost+ $60 \mathrm{~kg} \mathrm{~N}$ gave the highest average fruit weight in the first season

Table 10. Effect of interaction between nitrogen and organic, potassium and calcium on plant length and number of leaves.

\begin{tabular}{|c|c|c|c|c|c|c|c|c|c|}
\hline \multirow{4}{*}{$\begin{array}{c}\text { Compost } \\
\text { and } \\
\mathbf{N} \text { rates }\end{array}$} & \multicolumn{5}{|c|}{ Plant length $(\mathrm{m})$} & \multicolumn{4}{|c|}{ Number of leaves/plant } \\
\hline & \multicolumn{3}{|c|}{2011} & \multicolumn{2}{|c|}{2012} & 2011 & \multicolumn{3}{|c|}{2012} \\
\hline & \multicolumn{9}{|c|}{ Potassium level(kg/fed) } \\
\hline & $\begin{array}{l}\text { Calcium } \\
\text { level }(400 \mathrm{~g} / \mathrm{fed})\end{array}$ & 100 & 150 & 100 & 150 & 100 & 150 & 100 & 150 \\
\hline \multirow[t]{2}{*}{1} & unsprayed & $\begin{array}{l}1.51 \\
\mathrm{f}\end{array}$ & $1.52 \mathrm{f}$ & $1.68 \mathrm{i}$ & $1.79 \mathrm{hi}$ & 180.31 & $190.3 \mathrm{kl}$ & $191.3 \mathrm{~h}$ & $196.0 \mathrm{~h}$ \\
\hline & Sprayed & $\begin{array}{l}1.65 \\
\text { ef }\end{array}$ & 1.68 ef & $1.83 \mathrm{hi}$ & $\begin{array}{c}1.96 f g \\
h\end{array}$ & $199.3 \mathrm{ijk}$ & 193.3jkl & $\begin{array}{c}209.7 \mathrm{~d} \\
\mathrm{gh}\end{array}$ & $209.3 \mathrm{gh}$ \\
\hline \multirow[t]{2}{*}{2} & unsprayed & $\begin{array}{l}1.68 \\
\text { ef }\end{array}$ & $1.78 \mathrm{ef}$ & $1.95 \mathrm{fgh}$ & $\begin{array}{c}2.00 f g \\
h\end{array}$ & $\begin{array}{c}209.0 \\
\text { hij }\end{array}$ & 212ghi & $\begin{array}{c}212.0 \\
\text { gh }\end{array}$ & $224.7 \mathrm{fg}$ \\
\hline & Sprayed & $\begin{array}{l}1.76 \\
\text { ef }\end{array}$ & $\begin{array}{l}1.79 \\
\text { def }\end{array}$ & 1.90ghi & $\begin{array}{c}1.98 f g \\
h\end{array}$ & $\begin{array}{c}219.0 \mathrm{ef} \\
\mathrm{g}\end{array}$ & $\begin{array}{c}219.0 d- \\
h\end{array}$ & $\begin{array}{c}224.3 \\
\mathrm{fg}\end{array}$ & $\begin{array}{c}230.0 \\
\text { efg }\end{array}$ \\
\hline \multirow[t]{2}{*}{3} & unsprayed & $\begin{array}{l}1.93 \\
\text { b-e }\end{array}$ & $\begin{array}{l}1.92 \mathrm{~b}- \\
\mathrm{e}\end{array}$ & $2.11 d-h$ & $\begin{array}{c}2.15 \mathrm{c}- \\
\mathrm{g}\end{array}$ & 218.fgh & $\begin{array}{c}225.3 d- \\
h\end{array}$ & $\begin{array}{c}228.7 \\
\text { efg }\end{array}$ & $234.3 \mathrm{ef}$ \\
\hline & Sprayed & $\begin{array}{l}1.97 \\
\text { b-e }\end{array}$ & $\begin{array}{l}2.11 \mathrm{a}- \\
\mathrm{d}\end{array}$ & $2.28 \mathrm{~b}-\mathrm{e}$ & $\begin{array}{c}2.31 \mathrm{~b}- \\
\mathrm{e}\end{array}$ & $\begin{array}{c}235.3 \mathrm{~cd} \\
\mathrm{e}\end{array}$ & $\begin{array}{c}238.7 b c \\
d\end{array}$ & $\begin{array}{c}241.7 \\
c-f\end{array}$ & $\begin{array}{c}258.0 \mathrm{a}- \\
\mathrm{d}\end{array}$ \\
\hline \multirow[t]{2}{*}{4} & unsprayed & $\begin{array}{l}2.15 \\
a b c\end{array}$ & $\begin{array}{l}2.11 \text { a- } \\
\text { d }\end{array}$ & $\begin{array}{c}2.36 b c \\
d\end{array}$ & $2.38 b c$ & $\begin{array}{c}248.7 a b \\
c\end{array}$ & $\begin{array}{c}249.0 \mathrm{ab} \\
\mathrm{c}\end{array}$ & $\begin{array}{c}256.0 a- \\
d\end{array}$ & $\begin{array}{c}260.3 a b \\
c\end{array}$ \\
\hline & Sprayed & $\begin{array}{l}2.25 \\
\mathrm{ab} \\
\end{array}$ & $2.36 \mathrm{a}$ & $2.42 \mathrm{ab}$ & $2.65 \mathrm{a}$ & $253.7 \mathrm{ab}$ & $261.7 \mathrm{a}$ & $\begin{array}{c}265.0 \mathrm{a} \\
\mathrm{b}\end{array}$ & $268.0 \mathrm{a}$ \\
\hline \multirow[t]{2}{*}{5} & unsprayed & $\begin{array}{l}1.80 \\
\text { def }\end{array}$ & $\begin{array}{l}1.89 \\
\text { cde }\end{array}$ & $2.09 \mathrm{efg}$ & $2.18 b-f$ & $\begin{array}{c}228.0 d- \\
\mathrm{g}\end{array}$ & 231.3def & $\begin{array}{c}237.3 \\
\text { def }\end{array}$ & $240.7 \mathrm{c}-\mathrm{f}$ \\
\hline & Sprayed & $\begin{array}{l}1.86 \\
\text { cde }\end{array}$ & $\begin{array}{l}1.92 \mathrm{~b}- \\
\mathrm{e}\end{array}$ & $2.15 \mathrm{c}-\mathrm{g}$ & $\begin{array}{c}2.11 \mathrm{~d}- \\
\mathrm{g}\end{array}$ & $\begin{array}{c}226.3 d- \\
g\end{array}$ & $\begin{array}{c}237.7 b c \\
d\end{array}$ & $\begin{array}{c}245.7 b- \\
\mathrm{e}\end{array}$ & $\begin{array}{c}241.7 \mathrm{c}- \\
\mathrm{f}\end{array}$ \\
\hline \multirow[t]{2}{*}{6} & unsprayed & $\begin{array}{l}1.89 \\
\text { cde }\end{array}$ & $\begin{array}{l}1.843 \mathrm{c} \\
-\mathrm{f}\end{array}$ & $1.93 \mathrm{f}-\mathrm{i}$ & $\begin{array}{c}1.96 f g \\
h\end{array}$ & 193.3jkl & $220.3 e-h$ & $\begin{array}{c}233.0 \\
\text { ef }\end{array}$ & $234.0 \mathrm{ef}$ \\
\hline & Sprayed & $\begin{array}{l}1.92 \\
b-e\end{array}$ & $\begin{array}{l}1.897 \mathrm{c} \\
-\mathrm{e}\end{array}$ & $1.99 \mathrm{fgh}$ & $\begin{array}{c}2.02 \mathrm{fg} \\
\mathrm{h}\end{array}$ & $\begin{array}{c}217.7 \mathrm{fg} \\
\mathrm{h}\end{array}$ & $\begin{array}{c}222.3 \mathrm{~d}- \\
\mathrm{h}\end{array}$ & $\begin{array}{l}235.0 \\
\text { ef }\end{array}$ & $\begin{array}{c}238.0 \\
\text { def }\end{array}$ \\
\hline L.S.D(0.05) & & \multicolumn{2}{|c|}{0.33} & \multicolumn{2}{|c|}{0.25} & \multicolumn{2}{|c|}{16.37} & \multicolumn{2}{|c|}{20.92} \\
\hline $\begin{array}{r}\text { Compost and } \\
1=(5 \text { ton } c \\
4=(2 \text { ton c }\end{array}$ & $\begin{array}{l}\text { oost + zero N } \\
\text { post }+60 \mathrm{~kg} \mathrm{~N}\end{array}$ & & $\begin{array}{l}2=(4 \\
5=(1\end{array}$ & $\begin{array}{l}\text { compo } \\
\text { compo }\end{array}$ & $\begin{array}{l}20 \mathrm{~kg} \mathrm{~N}) \\
80 \mathrm{~kg} \mathrm{~N}\end{array}$ & ed & $\begin{array}{l}(3 \text { ton } \mathrm{c} \\
\text { (zero to }\end{array}$ & $\begin{array}{l}\text { st }+4 \\
\text { npost }\end{array}$ & $\begin{array}{l}\text { N) /fed } \\
0 \mathrm{~kg} \mathrm{~N} \text { ) }\end{array}$ \\
\hline
\end{tabular}


Table 11. Effect of interaction between nitrogen and organic ,potassium and calcium on plant fresh and dry weight

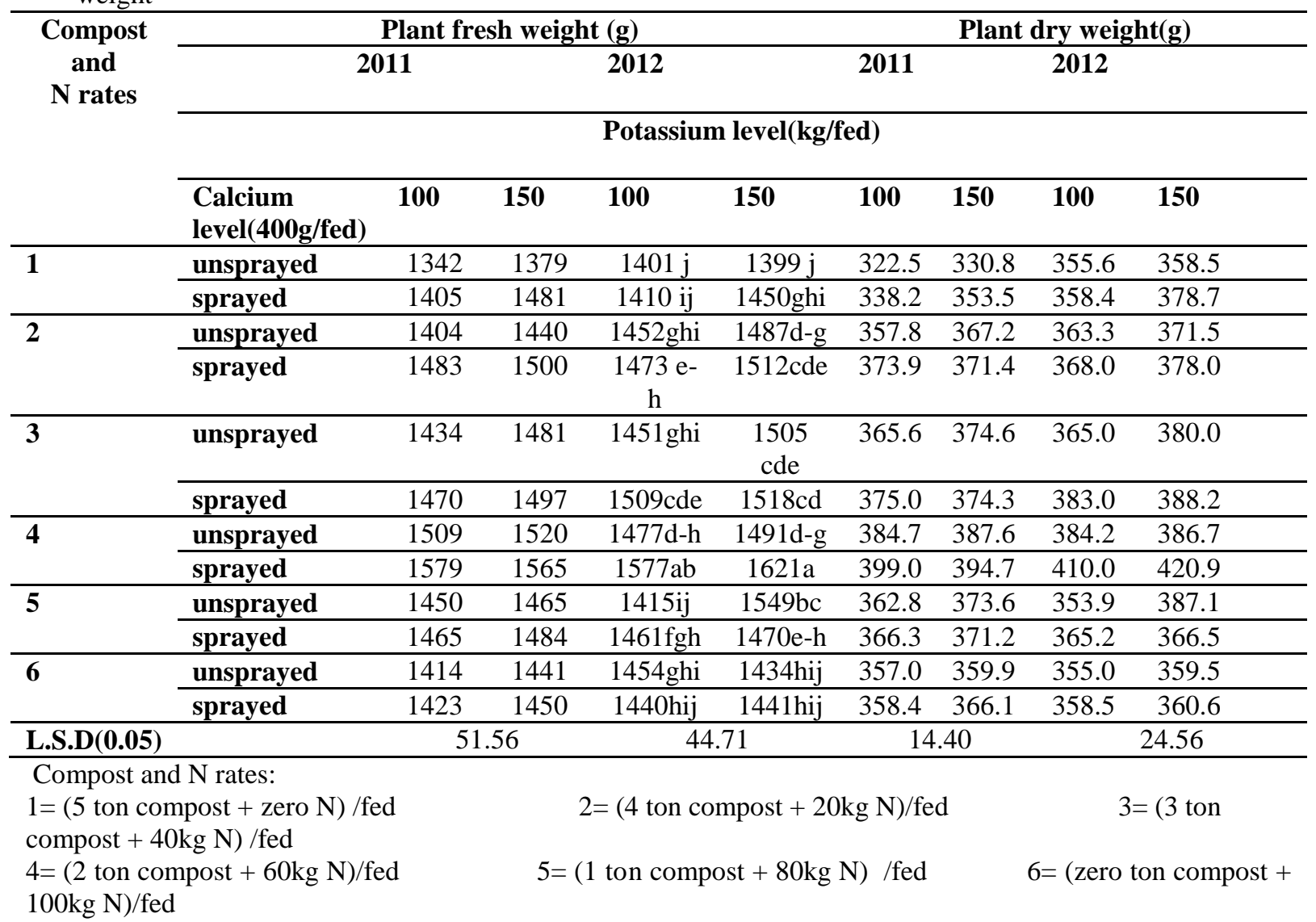

Table 12.Effect of organic and mineral, potassium and calcium on number of fruits and average fruit weight.

\begin{tabular}{|c|c|c|c|c|c|}
\hline \multirow[b]{2}{*}{ Treatments } & & \multicolumn{2}{|c|}{ Number of fruits/ plant } & \multicolumn{2}{|c|}{ Average fruit weight $/ \mathrm{kg}$} \\
\hline & & Season 2011 & $\begin{array}{c}\text { Season } \\
2012\end{array}$ & Season 2011 & Season 2012 \\
\hline \multirow{6}{*}{$\begin{array}{l}\text { Compost and } \\
\mathbf{N} \text { rates }\end{array}$} & 1 & $3.22 \mathrm{~d}$ & $3.27 \mathrm{~d}$ & $1.790 \mathrm{e}$ & $1.681 \mathrm{c}$ \\
\hline & 2 & $3.30 \mathrm{~d}$ & $3.48 \mathrm{c}$ & $1.918 \mathrm{~cd}$ & $1.794 \mathrm{bc}$ \\
\hline & 3 & $3.54 \mathrm{c}$ & $3.76 \mathrm{~b}$ & $2.047 \mathrm{ab}$ & $1.981 \mathrm{~b}$ \\
\hline & 4 & $4.22 \mathrm{a}$ & $4.26 \mathrm{a}$ & $2.157 \mathrm{a}$ & $2.375 \mathrm{a}$ \\
\hline & 5 & $3.68 \mathrm{~b}$ & $3.43 \mathrm{~cd}$ & $2.006 \mathrm{bc}$ & $2.013 \mathrm{~b}$ \\
\hline & 6 & $3.50 \mathrm{c}$ & $3.53 \mathrm{c}$ & $1.857 \mathrm{de}$ & $1.901 \mathrm{bc}$ \\
\hline L.S.D & & 0.13 & 0.18 & 0.115 & 0.220 \\
\hline Potassium & 100 & $3.50 \mathrm{~b}$ & $3.45 \mathrm{~b}$ & $1.916 \mathrm{~b}$ & $1.911 \mathrm{~b}$ \\
\hline Levels (kg/fed) & 150 & $3.65 \mathrm{a}$ & $3.79 \mathrm{a}$ & $2.009 \mathrm{a}$ & $2.004 \mathrm{a}$ \\
\hline L.S.D & & 0.06 & 0.10 & 0.066 & 0.085 \\
\hline \multirow{2}{*}{$\begin{array}{c}\text { Calcium levels } \\
(400 \mathrm{~g} / \mathrm{fed})\end{array}$} & unsprayed & $3.49 \mathrm{~b}$ & $3.54 \mathrm{~b}$ & $1.928 \mathrm{~b}$ & $1.927 \mathrm{~b}$ \\
\hline & sprayed & $3.66 \mathrm{a}$ & $3.70 \mathrm{a}$ & $1.997 \mathrm{a}$ & $1.987 \mathrm{a}$ \\
\hline L.S.D $(0.05)$ & & 0.06 & 0.06 & 0.053 & 0.055 \\
\hline $\begin{array}{l}\text { Compost and } \mathbf{N} \text { ra } \\
1=(5 \text { ton compost } \\
4=(2 \text { ton compost }\end{array}$ & $\begin{array}{l}: \\
\operatorname{akg~N} \text { )/fed }\end{array}$ & $\begin{array}{l}2=(4 \text { ton } c \\
5=(1 \text { ton } c\end{array}$ & $\begin{array}{l}\text { ost }+20 \mathrm{k} \\
\mathrm{st}+80 \mathrm{~kg}\end{array}$ & $\begin{array}{l}3=(3 \text { tor } \\
6=(\text { zero }\end{array}$ & $\begin{array}{l}+40 \mathrm{~kg} \mathrm{~N}) / \mathrm{fed} \\
\mathrm{st}+100 \mathrm{~kg} \mathrm{~N}) / \mathrm{fed}\end{array}$ \\
\hline
\end{tabular}

\section{Effect of potassium level}

As shown in Table 12 the higher values for number of fruit and average fruit weight were recorded with $150 \mathrm{~kg} \mathrm{~K}_{2} \mathrm{O}$ than the other treatment in the both seasons

Effect of foliar calcium application

Data in Table 12 show that the foliar calcium application had significant effect on fruit number and average fruit weight in both seasons. The highest average fruit weights were recorded with foliar calcium application compared to unsprayed plants.

Effect of the interaction between organic and mineral and potassium level 
Regarding the effect of the interaction between organic and mineral and potassium level, the highest values of number of fruits and average fruit were recorded with 2 ton compost $+60 \mathrm{~kg} \mathrm{~N}$ with both levels of potassium, 3 ton compost $+40 \mathrm{~kg} \mathrm{~N}$ with 100 and $150 \mathrm{~kg} \mathrm{~K}_{2} \mathrm{O}$ and 1 ton compost $+80 \mathrm{~kg}$ with 150 $\mathrm{kg} \mathrm{K}_{2} \mathrm{O}$ in the first season gave the highest average fruit weight in the first season. While in the second one the treatment 2 ton compost $+60 \mathrm{~kg} \mathrm{~N}$ with $150 \mathrm{~kg} \mathrm{~K}_{2} \mathrm{O}$ gave the highest number of fruits and average fruit weight.

Table 13. Effect of the interaction between organic and mineral and potassium level on number of fruits and average fruit weight.

\begin{tabular}{|c|c|c|c|c|c|c|c|c|}
\hline \multirow{4}{*}{$\begin{array}{l}\text { Compost and } \\
\mathrm{N} \text { rates }\end{array}$} & \multicolumn{4}{|c|}{ Number of fruits /plant } & \multicolumn{4}{|c|}{ Average fruit weight / kg } \\
\hline & \multicolumn{2}{|c|}{2011} & \multicolumn{2}{|c|}{2012} & \multicolumn{2}{|c|}{2011} & \multicolumn{2}{|c|}{2012} \\
\hline & \multicolumn{8}{|c|}{ Potassium levels(kg/fed) } \\
\hline & 100 & 150 & 100 & 150 & 100 & 150 & 100 & 150 \\
\hline 1 & $3.17 \mathrm{f}$ & 3.27 ef & $3.24 \mathrm{f}$ & $3.29 \mathrm{f}$ & $1.715 \mathrm{e}$ & $1.865 \mathrm{de}$ & $1.722 \mathrm{fg}$ & $1.638 \mathrm{~g}$ \\
\hline 2 & $3.16 \mathrm{f}$ & $3.45 \mathrm{~cd}$ & 3.42 ef & $3.54 \mathrm{de}$ & $1.905 \mathrm{bcd}$ & $1.930 \mathrm{bcd}$ & $1.755 \mathrm{efg}$ & $1.833 \mathrm{~d}-\mathrm{g}$ \\
\hline 3 & $3.48 \mathrm{~cd}$ & $3.59 \mathrm{c}$ & $3.56 \mathrm{~cd}$ & $3.95 \mathrm{~b}$ & $2.036 a b c$ & $2.058 \mathrm{ab}$ & $1.953 \mathrm{~b}-\mathrm{e}$ & $2.008 \mathrm{bcd}$ \\
\hline 4 & $4.23 \mathrm{a}$ & $4.20 \mathrm{a}$ & $4.08 \mathrm{~b}$ & $4.44 \mathrm{a}$ & $2.151 \mathrm{a}$ & $2.163 \mathrm{a}$ & $2.095 \mathrm{~b}$ & $2.655 \mathrm{a}$ \\
\hline 5 & $3.56 \mathrm{c}$ & $3.80 \mathrm{~b}$ & $3.00 \mathrm{~g}$ & $3.870 \mathrm{bc}$ & $1.868 \mathrm{de}$ & $2.148 \mathrm{a}$ & $2.060 \mathrm{bc}$ & $1.965 \mathrm{bcd}$ \\
\hline 6 & $3.39 \mathrm{de}$ & $3.60 \mathrm{c}$ & 3.40 ef & $3.67 \mathrm{~cd}$ & $1.823 \mathrm{de}$ & $1.890 \mathrm{~cd}$ & $1.878 \mathrm{c}-\mathrm{f}$ & $1.925 b-f$ \\
\hline L.S.D(0.05) & \multicolumn{2}{|c|}{0.15} & \multicolumn{2}{|c|}{0.24} & \multicolumn{2}{|c|}{0.164} & \multicolumn{2}{|c|}{0.210} \\
\hline \multicolumn{9}{|c|}{$\begin{array}{l}\text { Compost and } \mathrm{N} \text { rates: } \\
1=(5 \text { ton compost }+ \text { zero } \mathrm{N}) / \mathrm{fed} \\
4=(2 \text { ton compost }+60 \mathrm{~kg} \mathrm{~N}) / \mathrm{fed}\end{array}$} \\
\hline
\end{tabular}

Effect of the interaction between organic and mineral and calcium foliar application

With respect to the effect of the interaction between organic and mineral and calcium foliar application, data in Table 14 show that the treatment of 2 ton compost $+60 \mathrm{~kg} \mathrm{~N}$ with calcium treatment gave the highest values of number of fruits and average fruit weight in the first season and also 2 ton compost $+60 \mathrm{~kg} \mathrm{~N}$ with unsprayed plants, 3 ton compost $+40 \mathrm{~kg} \mathrm{~N}$ with sprayed calcium gave the highest average fruit weight in the first season. also 3 ton compost $+80 \mathrm{~kg} \mathrm{~N}, 1$ ton compost $+80 \mathrm{~kg} \mathrm{~N}$ with calcium foliar application gave the highest average fruit weight in the first seasons.

Table 14. Effect of the interaction between organic and mineral and calcium on fruit number and average fruit weight

\begin{tabular}{|c|c|c|c|c|c|c|c|c|}
\hline \multirow{4}{*}{$\begin{array}{l}\text { Compost } \\
\text { and } \\
\mathrm{N} \text { rates }\end{array}$} & \multicolumn{4}{|c|}{ Number of fruits /plant } & \multicolumn{4}{|c|}{ Average fruit weight / kg } \\
\hline & \multicolumn{2}{|c|}{2011} & \multicolumn{2}{|c|}{2012} & \multicolumn{2}{|c|}{2011} & \multicolumn{2}{|c|}{2012} \\
\hline & \multicolumn{8}{|c|}{ Calcium levels(400g/fed) } \\
\hline & unsprayed & sprayed & unsprayed & sprayed & unsprayed & sprayed & unsprayed & Sprayed \\
\hline 1 & $3.19 \mathrm{f}$ & $3.25 \mathrm{ef}$ & $3.29 \mathrm{f}$ & $3.31 \mathrm{f}$ & $1.770 \mathrm{e}$ & $1.81 \mathrm{de}$ & $1.705 \mathrm{gh}$ & $1.656 \mathrm{~h}$ \\
\hline 2 & $3.20 \mathrm{f}$ & $3.41 \mathrm{de}$ & $3.24 \mathrm{f}$ & $3.7 \mathrm{bcd}$ & $1.907 \mathrm{~cd}$ & $1.928 \mathrm{~cd}$ & $1.789 \mathrm{fgh}$ & $1.799 \mathrm{efg}$ \\
\hline 3 & $3.51 \mathrm{~d}$ & $3.56 \mathrm{~d}$ & $3.75 \mathrm{bc}$ & $3.77 \mathrm{~b}$ & $1.991 \mathrm{bc}$ & $2.104 \mathrm{ab}$ & $1.942 \mathrm{~cd}$ & $2.020 \mathrm{bc}$ \\
\hline 4 & $4.12 \mathrm{~b}$ & $4.31 \mathrm{a}$ & $4.24 \mathrm{a}$ & $4.27 \mathrm{a}$ & $2.140 \mathrm{a}$ & $2.174 \mathrm{a}$ & $2.320 \mathrm{a}$ & $2.430 \mathrm{a}$ \\
\hline 5 & $3.46 \mathrm{~d}$ & $3.900 \mathrm{c}$ & $3.30 \mathrm{f}$ & $3.57 \mathrm{de}$ & $1.908 \mathrm{~cd}$ & $2.103 \mathrm{ab}$ & $1.940 \mathrm{~cd}$ & $2.086 \mathrm{~b}$ \\
\hline 6 & $3.47 \mathrm{~d}$ & $3.52 \mathrm{~d}$ & $3.47 \mathrm{de}$ & $3.60 \mathrm{~cd}$ & $1.850 \mathrm{de}$ & $1.863 \mathrm{cde}$ & $1.868 \mathrm{def}$ & $1.934 \mathrm{cde}$ \\
\hline L.S.D $(0.05)$ & \multicolumn{2}{|c|}{0.16} & \multicolumn{2}{|c|}{0.16} & \multicolumn{2}{|c|}{0.130} & \multicolumn{2}{|c|}{0.135} \\
\hline \multicolumn{3}{|c|}{ Compost and $\mathrm{N}$ rates: } & & $\begin{array}{l}\text { kg N)/fed } \\
\mathrm{kg} \mathrm{N} \text { )/fed }\end{array}$ & & $\begin{array}{l}3 \text { ton compo } \\
\text { ero ton com }\end{array}$ & $\begin{array}{l}40 \mathrm{~kg} \mathrm{~N}) / \mathrm{fed} \\
+100 \mathrm{~kg} \mathrm{~N}) /\end{array}$ & \\
\hline
\end{tabular}

\section{Effect of the interaction between potassium and} calcium foliar application

It is clear from data in Table 21 that the highest values of number of fruits and average fruit weight were recorded when plants fertilized by $150 \mathrm{~kg} \mathrm{~K} 2 \mathrm{O}$ and treated with calcium at $400 \mathrm{~g} / \mathrm{fed}$ in both seasons. And also the plants that fertilized by $150 \mathrm{~kg} \mathrm{~K} 2$ Oand unsprayed with calcium gave the highest average fruit weight in the second season.
Effect of the interaction between organic and mineral, potassium level and calcium foliar application

The triple interaction between organic and mineral, potassium and calcium foliar application had a significant effect on the number of fruits in both seasons. Data in Table 16 show that the highest number of fruits were recorded when plants fertilized by 2 ton compost $+60 \mathrm{~kg} \mathrm{~N}$ with 100 and $150 \mathrm{~kg}$ $\mathrm{K}_{2} \mathrm{O}$ and sprayed with $400 \mathrm{~g}$ calcium /fed in both seasons and also 2 ton compost $+60 \mathrm{~kg} \mathrm{~N}$ with 100 
and $150 \mathrm{~kg} \mathrm{~K}_{2} \mathrm{O}$ and unsprayed plants in the first season .

While the highest average fruit weight was recorded with 1 ton compost $+80 \mathrm{~kg} \mathrm{~N}$ that fertilized by $150 \mathrm{~kg} \mathrm{~K}_{2} \mathrm{O}$ and sprayed with calcium in the first season. But in the second season the treatment with 2 ton compost+ $60 \mathrm{~kg} \mathrm{~N}$ and fertilized by $150 \mathrm{~kg} \mathrm{~K}_{2} \mathrm{O}$ and sprayed plants with calcium.

Table 15. Effect of the interaction between potassium and calcium foliar application on number of fruit and average fruit weight.

\begin{tabular}{|c|c|c|c|c|c|c|c|c|}
\hline \multirow{4}{*}{$\begin{array}{l}\text { Potassium } \\
\text { Level(kg/fed) }\end{array}$} & \multicolumn{4}{|c|}{ Number of fruits / plant } & \multicolumn{4}{|c|}{ Average fruit weight / kg } \\
\hline & \multicolumn{2}{|c|}{2011} & \multicolumn{2}{|c|}{2012} & \multicolumn{2}{|c|}{2011} & \multicolumn{2}{|c|}{2012} \\
\hline & \multicolumn{8}{|c|}{ Calcium level(400g/fed) } \\
\hline & unsprayed & sprayed & unsprayed & sprayed & unsprayed & sprayed & unsprayed & sprayed \\
\hline 100 & $3.42 \mathrm{c}$ & $3.58 \mathrm{~b}$ & $3.38 \mathrm{c}$ & $3.52 \mathrm{c}$ & $1.903 \mathrm{~b}$ & $1.928 \mathrm{~b}$ & $1.887 \mathrm{c}$ & $1.934 \mathrm{bc}$ \\
\hline 150 & $3.56 \mathrm{~b}$ & $3.74 \mathrm{a}$ & $3.69 \mathrm{~b}$ & $3.89 \mathrm{a}$ & $1.952 \mathrm{~b}$ & $2.066 \mathrm{a}$ & $1.967 \mathrm{ab}$ & $2.041 \mathrm{a}$ \\
\hline L.S.D(0.05) & \multicolumn{2}{|c|}{0.09} & \multicolumn{2}{|c|}{0.09} & \multicolumn{2}{|c|}{0.075} & \multicolumn{2}{|c|}{0.078} \\
\hline
\end{tabular}

Table 16. Effect of the interaction between organic and mineral, potassium and calcium foliar application on fruit number and average fruit weight

\begin{tabular}{|c|c|c|c|c|c|c|c|c|c|}
\hline \multirow{4}{*}{$\begin{array}{c}\text { Compost } \\
\text { and } \\
\mathrm{N} \text { rates }\end{array}$} & \multicolumn{5}{|c|}{ Number of fruits / plant } & \multicolumn{4}{|c|}{ Average fruit weight / kg } \\
\hline & \multicolumn{3}{|c|}{2011} & \multicolumn{2}{|c|}{2012} & \multicolumn{2}{|c|}{2011} & \multicolumn{2}{|c|}{2012} \\
\hline & \multicolumn{9}{|c|}{ Potassium level (kg/fed) } \\
\hline & $\begin{array}{c}\text { Calcium } \\
\text { level }\end{array}$ & 100 & 150 & 100 & 150 & 100 & 150 & 100 & 150 \\
\hline \multirow[t]{2}{*}{1} & Unsprayed & $3.14 \mathrm{~lm}$ & $3.20 \mathrm{klm}$ & $3.20 \mathrm{hi}$ & $3.29 \mathrm{gh}$ & $1.740 \mathrm{hi}$ & $1.690 \mathrm{i}$ & $1.750 \mathrm{~h}-\mathrm{k}$ & $1.695 \mathrm{ijk}$ \\
\hline & sprayed & $3.25 \mathrm{j}-\mathrm{m}$ & $3.30 \mathrm{i}-\mathrm{m}$ & $3.25 \mathrm{~h}$ & $3.32 \mathrm{gh}$ & $1.800 \mathrm{ghi}$ & $1.930 \mathrm{efg}$ & $1.660 \mathrm{jk}$ & $1.617 \mathrm{k}$ \\
\hline \multirow[t]{2}{*}{2} & Unsprayed & $3.08 \mathrm{~m}$ & $3.25 \mathrm{j}-\mathrm{m}$ & $3.27 \mathrm{gh}$ & $3.57 \mathrm{ef}$ & $1.900 \mathrm{e}-\mathrm{h}$ & $1.910 \mathrm{e}-\mathrm{h}$ & $1.750 \mathrm{~h}-\mathrm{k}$ & $1.760 \mathrm{~h}-\mathrm{k}$ \\
\hline & sprayed & $3.33 \mathrm{~h}-1$ & $3.57 \mathrm{~d}-\mathrm{g}$ & $3.22 \mathrm{hi}$ & $3.87 \mathrm{~cd}$ & $1.913 \mathrm{e}-\mathrm{h}$ & $1.947 \mathrm{e}-\mathrm{g}$ & $1.827 \mathrm{f}-\mathrm{j}$ & $1.838 \mathrm{f}-\mathrm{j}$ \\
\hline \multirow[t]{2}{*}{3} & Unsprayed & $3.45 \mathrm{e}-\mathrm{j}$ & $3.51 \mathrm{e}-\mathrm{i} \mathrm{i}$ & 3.63 ef & $3.50 \mathrm{fg}$ & $2.000 \mathrm{c}-\mathrm{f}$ & $2.073 b-e$ & $1.917 \mathrm{~d}-\mathrm{h}$ & $1.990 \mathrm{~d}-\mathrm{g}$ \\
\hline & sprayed & $3.5 \mathrm{efg}$ & $3.61 \mathrm{def}$ & $3.87 \mathrm{~cd}$ & $4.04 \mathrm{bc}$ & $1.981 \mathrm{c}-\mathrm{g}$ & $2.135 \mathrm{bcd}$ & $1.967 \mathrm{~d}-\mathrm{g}$ & $2.050 \mathrm{cde}$ \\
\hline \multirow[t]{2}{*}{4} & Unsprayed & $4.14 \mathrm{ab}$ & $4.33 \mathrm{a}$ & $4.4 \mathrm{bc}$ & $4.15 \mathrm{~b}$ & $2.140 \mathrm{a}-\mathrm{d}$ & $2.162 \mathrm{abc}$ & $2.090 \mathrm{~cd}$ & $2.100 \mathrm{~cd}$ \\
\hline & sprayed & $4.11 \mathrm{ab}$ & $4.30 \mathrm{a}$ & $4.48 \mathrm{a}$ & $4.40 \mathrm{a}$ & $2.140 \mathrm{a}-\mathrm{d}$ & $2.186 a b$ & $2.550 \mathrm{~b}$ & $2.760 \mathrm{a}$ \\
\hline \multirow[t]{2}{*}{5} & Unsprayed & $3.32 \mathrm{~h}-1$ & $3.80 \mathrm{~cd}$ & $3.00 \mathrm{i}$ & $3.00 \mathrm{i}$ & $1.840 \mathrm{f}-\mathrm{i}$ & $1.887 \mathrm{fgh}$ & $1.880 \mathrm{e}-\mathrm{i}$ & $2.240 \mathrm{c}$ \\
\hline & sprayed & $3.60 \mathrm{~d}-\mathrm{g}$ & $4.00 \mathrm{f}-\mathrm{k}$ & 3.60 ef & $4.14 \mathrm{~b}$ & $1.975 \mathrm{~d}-\mathrm{g}$ & $2.320 \mathrm{a}$ & $2.000 \mathrm{def}$ & $1.931 \mathrm{~d}-\mathrm{h}$ \\
\hline \multirow[t]{2}{*}{6} & Unsprayed & $3.40 \mathrm{f}-\mathrm{k}$ & $3.38 \mathrm{~g}-\mathrm{k}$ & $3.20 \mathrm{hi}$ & $3.60 \mathrm{ef}$ & $1.800 \mathrm{gh} \mathrm{i}$ & $1.846 \mathrm{f}-\mathrm{i}$ & $1.937 \mathrm{~d}-\mathrm{h}$ & $1.818 \mathrm{f}-\mathrm{j}$ \\
\hline & sprayed & $3.55 \mathrm{e}-\mathrm{h}$ & $3.66 \mathrm{de}$ & $3.75 \mathrm{de}$ & $3.600 \mathrm{ef}$ & $1.900 \mathrm{e}-\mathrm{h}$ & 1.880fgh & $1.800 \mathrm{~g}-\mathrm{k}$ & $2.050 \mathrm{~cd} \mathrm{e}$ \\
\hline \multicolumn{2}{|l|}{ L.S.D (0.05) } & \multicolumn{2}{|c|}{0.23} & \multicolumn{2}{|c|}{0.23} & \multicolumn{2}{|c|}{0.184} & \multicolumn{2}{|c|}{0.192} \\
\hline $\begin{array}{l}\text { Compost and } \\
1=(5 \text { ton cor } \\
4=(2 \text { ton cor }\end{array}$ & $\mathrm{st}+60 \mathrm{~kg} \mathrm{I}$ & & \multicolumn{3}{|c|}{$\begin{array}{c}2=(4 \text { ton compost }+20 \mathrm{~kg} \mathrm{~N}) / \mathrm{fed} \\
5=(1 \text { ton compost }+80 \mathrm{~kg} \mathrm{~N}) / \mathrm{fed}\end{array}$} & \multicolumn{4}{|c|}{$\begin{array}{c}3=(3 \text { ton compost }+40 \mathrm{~kg} \mathrm{~N}) / \mathrm{fed} \\
6=(\text { zero ton compost }+100 \mathrm{~kg} \mathrm{~N}) / \mathrm{fed}\end{array}$} \\
\hline
\end{tabular}

Yield Effect of organic and mineral fertilization

Data presented in Table 17 indicate that the highest values of yield /plant and yield/fed were produced with 2 ton compost $+60 \mathrm{~kg} \mathrm{~N}$ in both seasons, while the lowest values of these parameters were recorded with zero ton compost $+100 \mathrm{~kg} \mathrm{~N}$ in both seasons. Coelho et al. (2001) reported that applying $\mathrm{N}$ levels through drip irrigation at $(0$, 60,120 and $180 \mathrm{~kg} / \mathrm{ha}$ ) increased the yield of melon and the level of $120 \mathrm{~kg} / \mathrm{h}$ was the most suitable for melon crop.

Fontes et al.(2004) studied the effect of five $\mathrm{N}$ rates $\left(0,75,150,300\right.$, and $\left.450 \mathrm{~kg} \mathrm{ha}^{-1}\right)$, as urea in greenhouse and in open field, on muskmelon the experimental results showed that at both conditions, total and marketable melon fruit yields increased with increasing $\mathrm{N}$ rates.. Similar results were found by Meena et al. ( 2005) who found that the increasing levels of $\mathrm{N}$ up to $60 \% \mathrm{~kg} / \mathrm{ha}$ significantly increased fruit and biomass yield of snap melon (Cucumis melo var.momordica).

The apparent deficiency of an adequate supply of plant-available $\mathrm{N}$ from organic fertilizer, resulting from a slow rate of mineralization, makes crop yields in fields treated with organic fertilizer lower than in those treated with chemical fertilizers (Blatt, 1991; Lee, 2010).

In this respect increase in the total yield resulting that organic manuring enhanced soil aggregation, soil areation and increasing water holding capacity and offers good environmental conditions for the root system ( Abou El- Magd ,2005.) 
Organic manures like compost discharge nutrients very slowly to the plants and these nutrients are not directly absorb by the plants. Therefore, plants are unable access required amount of nutrients in the critical yield-forming period. This may be the probable reason for the higher yield produced by the inorganic fertilizer applied. Thus, combination of organic and inorganic fertilizers could produce better yields than organic manure alone Seran et al. (2010)

The combination of organic materials with reduced NPK fertilizer rates produced plants that were similar to unmixed NPK fertilizer. This indicated that the high dose of organic manures can be reduced by half and mixed with a reduced rate of NPK fertilizers as reported by Akande et al. (2003).

Table 17. Effect of organic and mineral, potassium and calcium on yield

\begin{tabular}{|c|c|c|c|c|c|}
\hline \multirow{2}{*}{\multicolumn{2}{|c|}{ Treatments }} & \multirow{2}{*}{\multicolumn{2}{|c|}{ Yield Per/plant (kg) }} & \multirow{2}{*}{\multicolumn{2}{|c|}{ Yield Per/fed (ton) }} \\
\hline & & & & & \\
\hline & & \multirow{2}{*}{$\begin{array}{c}\text { Season } \\
2011 \\
577 \mathrm{~d}\end{array}$} & $\begin{array}{l}\text { Season } \\
2012\end{array}$ & Season 2011 & $\begin{array}{c}\text { Season } \\
2012 \\
\end{array}$ \\
\hline Compost and & 1 & & $5.49 \mathrm{~d}$ & $23.11 \mathrm{~d}$ & $23.10 \mathrm{e}$ \\
\hline \multirow[t]{5}{*}{$\mathrm{N}$ rates } & 2 & $6.35 \mathrm{c}$ & $6.25 \mathrm{~cd}$ & $25.40 \mathrm{c}$ & $25.00 \mathrm{~d}$ \\
\hline & 3 & $7.24 \mathrm{~b}$ & $7.46 \mathrm{~b}$ & $29.00 \mathrm{~b}$ & $30.22 \mathrm{~b}$ \\
\hline & 4 & $9.10 \mathrm{a}$ & $10.18 \mathrm{a}$ & $36.42 \mathrm{a}$ & $39.94 \mathrm{a}$ \\
\hline & 5 & $7.42 \mathrm{~b}$ & $6.89 \mathrm{bc}$ & $29.68 \mathrm{~b}$ & $27.42 \mathrm{c}$ \\
\hline & 6 & $6.50 \mathrm{c}$ & $6.71 \mathrm{bc}$ & $26.02 \mathrm{c}$ & $26.95 \mathrm{c}$ \\
\hline L.S.D & & 0.42 & 1.07 & 1.68 & 1.447 \\
\hline Potassium & 100 & $6.75 \mathrm{~b}$ & $6.61 \mathrm{~b}$ & $26.33 b$ & $27.09 \mathrm{~b}$ \\
\hline Levels (kg/fed) & 150 & $7.38 \mathrm{a}$ & $7.71 \mathrm{a}$ & $29.12 \mathrm{a}$ & $30.45 \mathrm{a}$ \\
\hline L.S.D & & 0.25 & 0.48 & 0.6465 & 1.227 \\
\hline Calcium & unsprayed & $6.77 \mathrm{~b}$ & $6.89 \mathrm{~b}$ & $27.09 \mathrm{~b}$ & $27.75 b$ \\
\hline levels $(400 \mathrm{~g} / \mathrm{fed})$ & sprayed & $7.36 \mathrm{a}$ & $7.43 \mathrm{a}$ & $29.45 \mathrm{a}$ & $29.80 \mathrm{a}$ \\
\hline L.S.D $(0.05)$ & & 0.25 & 0.10 & 1.030 & 0.7768 \\
\hline $\begin{array}{l}\text { Compost and N rates } \\
1=(5 \text { ton compost }+ \\
5=(1 \text { ton compost }+\end{array}$ & $\begin{array}{l}\text { ) /fed } \\
\text { v) /fed }\end{array}$ & $4=(2$ ton co & $\begin{array}{l}20 \mathrm{~kg} \mathrm{~N}) / \mathrm{fed} \\
50 \mathrm{~kg} \mathrm{~N}) / \mathrm{fed}\end{array}$ & $\begin{array}{l}3=(3 \text { ton compos } \\
6=(\text { zero ton comp }\end{array}$ & $\begin{array}{l}\mathrm{kg} \mathrm{N}) / \mathrm{fed} \\
100 \mathrm{~kg} \mathrm{~N}) / \mathrm{fed}\end{array}$ \\
\hline
\end{tabular}

\section{Effect of potassium levels on yield}

It appears from data in Table 17 that plants received $150 \mathrm{~kg} \mathrm{~K} \mathrm{~K}_{2} \mathrm{O}$ gave the higher yield/plant and yield/feddan than the other treatment of $100 \mathrm{~kg} \mathrm{~K}_{2} \mathrm{O}$ in both seasons.

\section{Effect of calcium application on yield}

Data in Table 17 show that plants treated with 400g calcium /fed produced the higher yield/plant and yield /fed than untreated plants.

Non significant differences were recorded on yield, number of fruits and average fruit weight due to increasing the rate of potassium . These results are in agreement with those reported by Lin et al. (2004) on muskmelon, Demiral and Köseoglu (2005). On galia melon. On the other hand Okur and Yacmur (2004) found that increasing the rate of potassium increased the fruit weight on water melon.

The effect of the interaction between organic and mineral and potassium level on yield

Regarding the effect of the interaction between organic and mineral fertilization and potassium levels, data in Table 18 show that the highest yield/plant and yield per/fed were recorded when plants received 2 ton compost $+60 \mathrm{~kg} \mathrm{~N}$ and fertilized by $150 \mathrm{~kg} \mathrm{~K} \mathrm{~K}_{2} \mathrm{O}$ in both seasons and also 2 ton compost $+60 \mathrm{~kg} \mathrm{~N}$ and fertilized by $100 \mathrm{~kg} \mathrm{~K}_{2} \mathrm{O}$ in the first season. but the lowest values of yield/plant and yield/fed were recorded with zero ton compost $+100 \mathrm{~kg} \mathrm{~N}$ and fertilized by $100 \mathrm{~kg} \mathrm{~K} 2 \mathrm{O}$ in both seasons .

Generally the significant increasing in the growth and yield as applied $\mathrm{N}$ rates increased confirmed the role of $\mathrm{N}$ in the promotion of vegetative growth and yield especially when applied at the recommended rate. (Olaniyi and Odedere, 2009).

This result is similar to the finding by Akanbi et al. (2005) and olaniyi (2006) who reported an increase in the performance fruit vegetable. The application of compost had significant effect on growth, yield and nutrient uptake possibly due to the ability of compost to support plant growth for an extended period of time, which makes essential nutrient available to crops over a long period. The $\mathrm{N}$ in compost is released slowly by microorganism through the process of mineralization. Hence compost need not be applied frequently and crops make use it more efficiently than mineral $\mathrm{N}$ fertilizer.

Makinde et al. (2007) reported that increasing melon growth and optimum yield with organomineral fertilizer rate at 4 t/ha or the application inorganic fertilizer at $41 \mathrm{~kg} \mathrm{~N}+20 \mathrm{~kg} \mathrm{P}$. The combination of farm-yard manure and inorganic fertilizer significantly influenced cucumber yield compared to farm-yard manure and fertilizer alone especially at higher rates of application. The increasing in yield of cucumber could be attributed to the fact that nutrients were more readily available 
when organic and inorganic fertilizers were combined

Table 18. Effect of interaction between organic and mineral and potassium level on yield

\begin{tabular}{|c|c|c|c|c|c|c|c|c|}
\hline \multirow{4}{*}{$\begin{array}{l}\text { Compost and } \\
\text { N rate }\end{array}$} & \multicolumn{4}{|c|}{ Yield Per/plant(kg) } & \multicolumn{4}{|c|}{ Yield Per/fed(ton) } \\
\hline & \multicolumn{2}{|c|}{2011} & \multicolumn{2}{|c|}{2012} & \multicolumn{2}{|c|}{2011} & \multicolumn{2}{|c|}{2012} \\
\hline & \multicolumn{8}{|c|}{ Potassium levels(kg/fed) } \\
\hline & 100 & 150 & 100 & 150 & 100 & 150 & 100 & 150 \\
\hline 1 & $5044 \mathrm{f}$ & $6.11 \mathrm{e}$ & $5.58 \mathrm{f}$ & $5.39 \mathrm{f}$ & $21.76 \mathrm{f}$ & $24.46 \mathrm{e}$ & $22.35 \mathrm{f}$ & $21.59 \mathrm{f}$ \\
\hline 2 & 6.03 ef & $6.66 \mathrm{de}$ & $6.00 \mathrm{ef}$ & $6.50 \mathrm{def}$ & $24.14 \mathrm{ef}$ & 26.66de & $24.01 \mathrm{ef}$ & $26.02 \mathrm{def}$ \\
\hline 3 & $7.10 \mathrm{~cd}$ & $7.39 \mathrm{c}$ & 6.97 cde & $7.96 \mathrm{bc}$ & $28.42 \mathrm{~cd}$ & $29.57 \mathrm{c}$ & $27.88 \mathrm{cde}$ & $31.86 \mathrm{bc}$ \\
\hline 4 & $9.11 \mathrm{a}$ & $9.09 \mathrm{a}$ & $8.55 \mathrm{~b}$ & $11.80 \mathrm{a}$ & $36.45 \mathrm{a}$ & $36.38 \mathrm{a}$ & $34.22 b$ & $47.19 \mathrm{a}$ \\
\hline 5 & $6.64 \mathrm{de}$ & $8.19 \mathrm{~b}$ & 6.19 ef & $7.59 \mathrm{bcd}$ & 26.59de & $32.77 b$ & 24.79 ef & $30.36 \mathrm{bcd}$ \\
\hline 6 & $6.18 \mathrm{f}$ & $6.82 \mathrm{~cd}$ & 6.37 ef & $7.06 \mathrm{cde}$ & $24.75 \mathrm{e}$ & $27.29 \mathrm{~cd}$ & $25.49 \mathrm{ef}$ & $28.24 \mathrm{cde}$ \\
\hline L.S.D(0.05) & & & & & & & & \\
\hline
\end{tabular}

\section{Compost and $\mathrm{N}$ rates:}

$1=(5$ ton compost + zero $\mathrm{N}) / \mathrm{fed}$

$4=(2$ ton compost $+60 \mathrm{~kg} \mathrm{~N}) / \mathrm{fed}$

$$
\begin{gathered}
2=(4 \text { ton compost }+20 \mathrm{~kg} \mathrm{~N}) / \mathrm{fed} \\
5=(1 \text { ton compost }+80 \mathrm{~kg} \mathrm{~N}) / \mathrm{fed}
\end{gathered}
$$

$$
\begin{aligned}
& 3=(3 \text { ton compost }+40 \mathrm{~kg} \mathrm{~N}) / \mathrm{fed} \\
& 6=(\text { zero ton compost }+100 \mathrm{~kg} \mathrm{~N}) / \mathrm{fed}
\end{aligned}
$$

Effect of the interaction between organic and mineral and calcium foliar application on yield

Concerning to the effect of the interaction between organic and mineral and calcium foliar application, data in Table (19) show that the highest yield/plant and yield /fed were recorded when plants received 2 ton compost $+60 \mathrm{~kg} \mathrm{~N}$ and sprayed with $400 \mathrm{~g}$ calcium/fed in both seasons. While the lowest values of yield /plant and yield/fed were recorded when plants fertilizes by zero ton compost $+100 \mathrm{~kg} \mathrm{~N}$

\begin{tabular}{|c|c|c|c|c|c|c|c|c|}
\hline \multirow{4}{*}{$\begin{array}{l}\text { Compost } \\
\text { and } \\
\mathrm{N} \text { rate }\end{array}$} & \multicolumn{4}{|c|}{ Yield Per/plant(kg) } & \multicolumn{4}{|c|}{ Yield Per/fed(ton) } \\
\hline & \multicolumn{2}{|c|}{2011} & \multicolumn{2}{|c|}{2012} & \multicolumn{2}{|c|}{2011} & \multicolumn{2}{|c|}{2012} \\
\hline & \multicolumn{8}{|c|}{ Calcium levels (400g/fed) } \\
\hline & unsprayed & sprayed & unsprayed & sprayed & unsprayed & sprayed & unsprayed & Sprayed \\
\hline 1 & $5.65 \mathrm{~h}$ & $5.89 \mathrm{gh}$ & $5.50 \mathrm{e}$ & $5.48 \mathrm{e}$ & $22.63 \mathrm{~g}$ & $23.59 \mathrm{fg}$ & $22.01 \mathrm{e}$ & $21.93 \mathrm{e}$ \\
\hline 2 & $6.11 \mathrm{~g}$ & $6.58 \mathrm{f}$ & $5.80 \mathrm{e}$ & $6.70 \mathrm{~d}$ & 24.46 efg & 26.34de & $23.22 \mathrm{e}$ & $26.81 \mathrm{~d}$ \\
\hline 3 & $6.99 \mathrm{e}$ & $7.50 \mathrm{~d}$ & $7.29 \mathrm{bc}$ & $7.64 \mathrm{~b}$ & $27.97 \mathrm{~cd}$ & $30.02 \mathrm{c}$ & 29.18 bc & $30.56 \mathrm{~b}$ \\
\hline 4 & $8.82 \mathrm{~b}$ & $9.38 \mathrm{a}$ & $9.90 \mathrm{a}$ & $10.45 \mathrm{a}$ & $35.30 \mathrm{ab}$ & $37.53 \mathrm{a}$ & $36.62 \mathrm{a}$ & $41.79 \mathrm{a}$ \\
\hline 5 & $6.61 \mathrm{f}$ & $8.22 \mathrm{c}$ & $6.41 \mathrm{~d}$ & $7.37 \mathrm{bc}$ & $26.46 \mathrm{de}$ & $32.90 \mathrm{~b}$ & $26.67 \mathrm{~d}$ & $29.49 b c$ \\
\hline 6 & $6.43 \mathrm{f}$ & $6.157 \mathrm{f}$ & $6.47 \mathrm{~d}$ & 6.96 & 25.74 def & $26.3 \mathrm{de}$ & $25.89 \mathrm{~d}$ & $27.84 \mathrm{~cd}$ \\
\hline L.S.D(0.05) & \multicolumn{2}{|c|}{0.25} & \multicolumn{2}{|c|}{0.58} & \multicolumn{2}{|c|}{2.52} & \multicolumn{2}{|c|}{2.35} \\
\hline \multicolumn{9}{|c|}{$\begin{array}{l}\text { Compost and N rates: } \\
1=(5 \text { ton compost }+ \text { zero N }) / \text { fed } \\
4=(2 \text { ton compost }+60 \mathrm{~kg} \mathrm{~N}) / \mathrm{fed}\end{array}$} \\
\hline \multicolumn{9}{|c|}{$\begin{array}{l}\text { Effect of the interaction between potassium and } \\
\text { calcium foliar application on yield } \\
\text { It is clear from data presented in Table } 20\end{array}$} \\
\hline \multirow{4}{*}{$\begin{array}{c}\text { Potassium } \\
\text { Level(kg/fed) }\end{array}$} & \multicolumn{4}{|c|}{ Yield Per/plant(kg) } & \multicolumn{4}{|c|}{ Yield Per/fed(ton) } \\
\hline & \multicolumn{2}{|c|}{2011} & 2012 & & 2011 & & 2012 & \\
\hline & \multicolumn{8}{|c|}{ Calcium level $(400 \mathrm{~g} / \mathrm{fed})$} \\
\hline & Unsprayed & Sprayed & unsprayed s & prayed & unsprayed & sprayed & unsprayed & sprayed \\
\hline 100 & $6.55 \mathrm{c}$ & $6.95 \mathrm{~b}$ & $6.41 \mathrm{~d}$ & $5.81 \mathrm{c}$ & $26.23 \mathrm{c}$ & $27.80 \mathrm{~b}$ & $25.67 \mathrm{~d}$ & $27.25 \mathrm{c}$ \\
\hline \multirow[t]{2}{*}{$150(0.05)$} & $6.98 \mathrm{~b}$ & $7.77 \mathrm{a}$ & $7.38 \mathrm{~b}$ & $.057 \mathrm{a}$ & $27.95 \mathrm{~b}$ & $31.09 \mathrm{a}$ & $29.52 \mathrm{~b}$ & $32.23 \mathrm{a}$ \\
\hline & \multicolumn{2}{|c|}{0.36} & \multicolumn{2}{|c|}{0.33} & \multicolumn{2}{|c|}{1.45} & \multicolumn{2}{|l|}{1.35} \\
\hline
\end{tabular}
with sprayed and unsprayed with calcium in both seasons.

Table 19. Effect of the interaction between organic and mineral and calcium foliar application on yield 
Effect of the interaction between organic and mineral, potassium level and calcium foliar application on yield

Regarding to the effect of the interaction between organic and mineral, potassium level and calcium foliar application, data in Table 21 show that the highest values of yield/plant and yield/fed .were recorded with 2 ton compost $+60 \mathrm{~kg} \mathrm{~N}$ and fertilized with 100 and $150 \mathrm{~kg} \mathrm{~K}_{2} \mathrm{O}$ and sprayed with $400 \mathrm{~g}$ calcium /fed). On the other hand, the treatment with zero ton compost $+100 \mathrm{~kg} \mathrm{~N}$ with $100 \mathrm{~kg} \mathrm{~K}_{2} \mathrm{O}$ and unsprayed with calcium gave the lowest values of yield/plant and yield/fed in the first season .while the lowest values of yield/plant and yield/fed were recorded with 5ton compost + zero $\mathrm{N}$ with100 and $150 \mathrm{~K}_{2} \mathrm{O}$ and unsprayed with calcium

Table 21. Effect of the interaction between organic and mineral ,potassium and calcium foliar application yield/plant and yield/fed

\begin{tabular}{|c|c|c|c|c|c|c|c|c|c|}
\hline \multirow{4}{*}{$\begin{array}{c}\text { Compost and } \\
\text { N rate }\end{array}$} & \multicolumn{5}{|c|}{ Yield Per/plant (kg) } & \multicolumn{4}{|c|}{ Yield Per/fed(ton) } \\
\hline & \multicolumn{3}{|c|}{2011} & \multicolumn{2}{|c|}{2012} & \multicolumn{2}{|c|}{2011} & \multicolumn{2}{|c|}{2012} \\
\hline & \multicolumn{9}{|c|}{ Potassium level (kg/fed) } \\
\hline & $\begin{array}{c}\text { Calcium } \\
\text { level }(\mathrm{g} / \mathrm{fed})\end{array}$ & 100 & 150 & 100 & 150 & 100 & 150 & 100 & 150 \\
\hline \multirow{2}{*}{1} & Unsprayed & $5.46 \mathrm{j}$ & $5.41 \mathrm{j}$ & $5.59 \mathrm{ij}$ & $5.58 \mathrm{ij}$ & $21.85 \mathrm{j}$ & $21.67 \mathrm{j}$ & $22.38 \mathrm{ij}$ & $22.32 \mathrm{ij}$ \\
\hline & Sprayed & $5.85 \mathrm{ij}$ & $6.37 \mathrm{~d}-\mathrm{i}$ & $5.41 \mathrm{j}$ & $5.38 \mathrm{j}$ & $23.41 \mathrm{ij}$ & $25.51 \mathrm{~d}-\mathrm{i}$ & $21.64 \mathrm{i}$ & $21.51 \mathrm{j}$ \\
\hline \multirow{2}{*}{2} & Unsprayed & 5.86 hij & $6.20 \mathrm{f}-\mathrm{j}$ & $5.72 \mathrm{hij}$ & $6.28 \mathrm{ghi}$ & $23.46 \mathrm{~h}-\mathrm{j}$ & $24.82 \mathrm{f}-\mathrm{j}$ & 22.90hij & 25.13hij \\
\hline & Sprayed & $6.36 \mathrm{~d}-\mathrm{i}$ & $6.96 \mathrm{~b}-\mathrm{g}$ & 5.88 hij & $7.12 \mathrm{ef}$ & $25.46 \mathrm{~d}-\mathrm{i}$ & $27.85 \mathrm{~b}-\mathrm{g}$ & 23.54hij & $28.49 \mathrm{ef}$ \\
\hline \multirow{2}{*}{3} & Unsprayed & $6.92 \mathrm{~b}-\mathrm{g}$ & $7.28 \mathrm{bc}$ & $6.96 \mathrm{efg}$ & 6.97 efg & $27.70 \mathrm{~b}-\mathrm{g}$ & $29.15 \mathrm{bc}$ & $27.88 \mathrm{efg}$ & $27.89 \mathrm{efg}$ \\
\hline & Sprayed & $7.06 \mathrm{~b}-\mathrm{f}$ & $7.72 \mathrm{~b}$ & 7.6 cde & $8.31 \mathrm{bc}$ & $28.24 b-f$ & $30.90 \mathrm{~b}$ & $30.48 \mathrm{cde}$ & $33.24 b c$ \\
\hline \multirow{2}{*}{4} & Unsprayed & $8.85 \mathrm{a}$ & $9.37 \mathrm{a}$ & $8.37 b c$ & $8.73 \mathrm{~b}$ & $35.42 \mathrm{a}$ & $37.49 \mathrm{a}$ & $33.50 b c$ & $34.94 b$ \\
\hline & Sprayed & $8.79 \mathrm{a}$ & $9.39 \mathrm{a}$ & $11.44 \mathrm{a}$ & $12.16 \mathrm{a}$ & $35.18 \mathrm{a}$ & $37.57 \mathrm{a}$ & $45.74 \mathrm{a}$ & $48.64 a$ \\
\hline \multirow{2}{*}{5} & unsprayed & $6.12 \mathrm{~g}-\mathrm{i}$ & $7.17 \mathrm{bcd}$ & $5.64 \mathrm{ij}$ & $6.75 \mathrm{fg}$ & $24.49 \mathrm{~g}-\mathrm{i}$ & $28.70 \mathrm{bcd}$ & $48.64 \mathrm{ij}$ & $27.02 \mathrm{fg}$ \\
\hline & sprayed & $7.10 \mathrm{~b}-\mathrm{e}$ & $9.27 \mathrm{a}$ & $7.19 \mathrm{def}$ & $7.98 \mathrm{bcd}$ & $28.43 b-e$ & $37.11 \mathrm{a}$ & $28.77 \mathrm{def}$ & $31.96 \mathrm{bcd}$ \\
\hline \multirow{2}{*}{6} & unsprayed & $6.12 \mathrm{~g}-\mathrm{j}$ & $6.25 \mathrm{e}-\mathrm{j}$ & $6.20 \mathrm{~g}-\mathrm{j}$ & $6.54 \mathrm{fgh}$ & $24.49 \mathrm{~g}-\mathrm{i}$ & $25.01 \mathrm{e}-\mathrm{j}$ & $24.80 \mathrm{~g}-\mathrm{j}$ & $26.19 \mathrm{fgh}$ \\
\hline & sprayed & $6.74 \mathrm{c}-\mathrm{h}$ & $6.89 \mathrm{~b}-\mathrm{g}$ & $6.74 \mathrm{fg}$ & $7.37 \mathrm{def}$ & $26.99 \mathrm{ch}$ & $27.59 \mathrm{~b}-\mathrm{g}$ & $26.98 \mathrm{fg}$ & $29.50 \mathrm{def}$ \\
\hline L.S.D & & \multicolumn{2}{|c|}{0.89} & \multicolumn{2}{|c|}{0.83} & \multicolumn{2}{|c|}{3.56} & \multicolumn{2}{|c|}{3.33} \\
\hline
\end{tabular}

Compost and $\mathrm{N}$ rates:

$1=(5$ ton compost + zero $\mathrm{N}) / \mathrm{fed} \quad 2=(4$ ton compost $+20 \mathrm{~kg} \mathrm{~N}) / \mathrm{fed} \quad 3=(3$ ton compost $+40 \mathrm{~kg} \mathrm{~N}) / \mathrm{fed}$

$4=(2$ ton compost $+60 \mathrm{~kg} \mathrm{~N}) / \mathrm{fed} \quad 5=(1$ ton compost $+80 \mathrm{~kg} \mathrm{~N}) /$ fed $\quad 6=($ zero ton compost $+100 \mathrm{~kg} \mathrm{~N}) / \mathrm{Fed}$

\section{References}

Abdalla,

A.A.G.;EL-Oksh,.I.I;

Abdallah,M.M.F.and EL-Sherif (2006).Effect of sugar beet compost and mineral nitrogen rates on cucumber yield grown in sandy soil .Egypt.J.Appl.sci.,21(8):694-709.

Abd El-Aziz, S.A. (2003). Studies on producing cantaloupe organically by using nutrient film technique (NFT). M.Sc. Thesis, Fac., Agric., Ain Shams Univ., Egypt, 9.

Abou El-Magd, M. M; Hoda, A. Mohammed and Fawzy, Z.F.( 2005). Relationship, growth and yield of broccoli with increasing $\mathrm{N}$, P or $\mathrm{K}$ ratio in a mixture of NPK fertilizers. Annals Agriculture Science Moshtohor, 43 (2):791-805

Abou EL-Nour, H .H. (2008) Effect of organic and biofertilization on growth and productivity of cantaloupe.Ph.D.Thesis,Fac.Agric.,Ain shams,Univ.,Egypt,188pp

Abou El-Yazied; A.I.I. El-Kassas;M. Abdalla and M.Z.M. El - Moslemany.(2012). Response of cantaloupe hybride to nitrogen fertilization levels under natural infection with $\mathrm{M}$ onosporascus cannonballus. Australian Journal of Basic and Applied Sciences .6 (3):1-11

Adam, S. M.; Abdalla, A.M. and F. A. Rizk,( 2002). Effect of interaction between the mineral and biofertilizer on the productivity of cantaloupe( Cucumis melo var L.) under newly reclaimed soil condition. . Egypt. J.Hort .29 (2):301-15.

Akanbi, W.B., Akande, M.O. and Adediran J.A. 2005. Suitability of composted maize straw and mineral nitrogen fertilizer for tomato production J. of Veg. Science, 11 (1): 57-65

Akande, M. O.; Oluwatoyinbo, F. I.; Adediran, J. A.; Buari, K. W. and Yusuf, I. O. (2003). Soil amendments affect the release of $\mathrm{P}$ from rock phosphate and the development and yield of okra. J of Veg. Crop Production,9(2):3-9.

Alvarez, C.E.; Garcia, C.and Carracedo A.E. (1988). Soil fertility and mineral nutrition of organic banana plantation in Tenerife. Biological Agriculture and Horticulture. 5: 313-323.

Arisha, H.M.E.; Gad, A.A. and Younes, S.E. (2003). Response of some pepper cultivars to organic and mineral nitrogen fertilizer under sandy soil conditions. Zagazig Journal Agriculture Research, 30: 1875-1899.

Bayoumi, Y.A. and Hafez, Y.M. (2006).Effect of organic fertilizers combined with benzo $(1,2,3)$ thiadiazole-7-carbothioic acid S-methyl ester ( BTH ) on the cucumber powdery mildew and the 
yield production. Acta Biologica Szegediensis, 50: 131-136.

Blatt, C.R. 1991. Comparison of several organic amendments with a chemical fertilizer for vegetable Production. Scientia Horticulturae. 47: 177-191.

Brady, N. C. (1990). The nature and properties of soil. pp. 512-515. New Delhi: Prentice Hall of India.

Castellanos, M. T.; Cabello, M. J.; Cartagena, M. D. C.; Tarquis, A. M., Arce, A. and Ribas, F. (2011). Growth dynamics and yield of melon as influenced by nitrogen fertilizer. Scientia Agricola, 68(2), 191-199.

Clark, M. S.; Horwath, W. R.; Shennan, C., and Scow, K. M. (1998). Changes in soil chemical properties resulting from organic and low-input farming practices. Agronomy Journal, 90(5), 662-671.

Coelho, E. F. ; Sousa, V. F.; de Souza, V. A. B.; de Melo, F. and de B. 2001. Effects of levels of $\mathrm{N}$ and $\mathrm{K}$ applied by drip irrigation on melon (Cucumis melo L.) grown on a sandy soil. Ciencia Agrotecnologia;. 25(1):23-30.

Cooke, G.W. (1972). Fertilizer for maximum yield. Richard clay (the Chaucer press LTD. Hungary, Suffolk. Britain. 457P Current Science. 79: 1716-1720

Demiral, M.; Köseoglu, A. (2005) .Effect of potassium on yield, fruit quality, and chemical composition of greenhouse-grown galia melon J. Plant Nutr. 93-100.

Farrag , D.K - (2009). Effect of organic and mineral fertilizers and biostimulants on growth,yield and quality of cantaloupe plant ( Cucumis melo var. cantaloupensis ,L).M.Sc. Thesis, Fac. Agric., Kafrelsheikh Univ., Egypt.

Dinesh, R.; Dubey, R.P.; Ganeshamurthy, A.N. and Prasad, G.S. (2000). Organic manuring in rice based cropping system: effects on soil microbial biomass and selected enzyme activities

Drinkwater, L.E.; Letourneau, D.K.; Workneh, F.; Bruggen, A.H.C. and Shennan, C. (1995). Fundamental difference between conventional and organic tomato agroecosystems in California. Ecological Applications. 5: 10981112

Eifediyi, E. K. and Remison, S. U. (2010). Growth and yield of cucumber (Cucumis sativus L.) as influenced by farmyard manure and inorganic fertilizer. Journal of Plant Breeding and Crop Science, 2(7), 216-220.

EL-Shafie and Eida E. ELGammaily.(2002).Effect of organic manure,sulphur and microelements on growth ,bulb yield,storability and chemical comoisition of onion plants.Minufiya J.Agic.Res.,27:407-424

Fontes, P. C. R.; Coelho, E. L.; Cardoso, A. A. and Finger, F. L. 2004. Muskmelon fruit yield in greenhouse and field conditions as affected by nitrogen rates.Bioscience Journal. 20(1):15-20.

Hafez, M.M. and Mahmoud ,A.R.(203).Respose of squash (Cucurbita pepo,L) to nitrogen source,nitrogen level and biofertilizer application.Annals Agric.Sc,Moshtohor,41:283298

Hesse PR, Mishra RV. (1982). Mineral on organic project field document no. 14 RAS/75/004FAO/UND project on organic recycling. FAO Roune, 114.

Jahromi, M. G.; Aboutalebi, A. and Farahi, M. H. (2012) . Influence of different levels of garden compost (garden wastes and cow manure) on growth and stand establishment of tomato and cucumber in greenhouse condition. Afr J Biotechnol, 11, 9036-9039

Jilani, M. S.; Bakar, A.; Waseem, K. A. S. H. I. F. and Kiran, M. (2009). Effect of different levels of NPK on the growth and yield of cucumber (Cucumis sativus) under the plastic tunnel. Journal of agriculture \& social sciences, 5, 99-101.

Lampkin, N. (1990). Organic farming. Farming press books. Ipswich. United Kingdom

Lee, J. 2010. Effect of application methods of organic fertilizer on growth, soil chemical properties and microbial densities in organic bulb onion production. Scientia Horticulturae. 124: 299-305

Lin, D., Huang, D., \& Wang, S. (2004). Effects of potassium levels on fruit quality of muskmelon in soilless medium culture. Scientia Horticulturae, 102(1), 53-60

Makinde, E. A., Ayoola, O. T. and Akande, M. O. (2007). Effects of organo-mineral fertilizer application on the growth and yield of egusi melon. Australian Journal of Basic and Applied Sciences, 1(1), 15-19.

Meena, K. R.; Verma, I. M. and Meena, R. P. (2005). Effect of different sowing environments and applied nitrogen on phenophase appearance, fruit yield and heat use efficiency of spring Sown Snapmelon (Cucumis melo var. momordica). Annals of Agri Bio Research, 10(2), 171.

Mohamed, M. A.E . (2012). Effect of some treatments on growth, yield and fruit chemical composition of melon and cucumber under sandy conditions.M.Sc. Thesis, Fac. Agric., Cairo. Univ., Egypt

Nafadi, M.H. and Gohar, H.O. (1975). Corn response to potassium as influenced by watering period, farmyard manure, sodium and ammonia solution and the residual effect on subsequent wheat. Egypt. J. Soil Sci., Special Issue, 307.

Okur, B., and Yagmur, B. (2004). Effects on enhanced potassium doses on yield, quality and nutrient uptake of watermelon. In IPI regional workshop on potassium and fertigation 
development in West Asia and North Africa.

Olaniyi, J.O. 2006. Influence of Nitrogen and Phosphorus fertilizer on seed yield and quality of Egusi melon [Citrullus lanatus (thunb) Mansf.], in Ogbomoso, Southwestern Nigeria. Ph.D Thesis, University of Ibadan, Ibadan, Nigeria. 199Pp.

Olaniyi, J. O., and Odedere, M. P. (2009). The effects of mineral $\mathrm{N}$ and compost fertilizers on the growth, yield and nutritional values of fluted pumpkin (Telfairia occidentalis) in south western Nigeria. J. Anim. Plant Sci, 5(1), 443449.

Olaniyi, J. O., Ogunbiyi, E. M.,and Alagbe, D. D. (2009). Effects of organo-mineral fertilizers on growth, yield and mineral nutrients uptake in cucumber. Journal of Animal \& Plant Sciences, 5(1), 437-442.

Peyvast, Gh.; Ramezani Kharazi, P.; Tahernia, S.; Nosratierad, Z.and Olfati, J.A. (2008). Municipal solid waste compost increased yield and decreased nitrate amount of broccoli (Brassica oleracea var. Italica). Journal of Applied Horticulture. 10(2): 129-13

Piper, C.S. (1950). Soil and Plant Analysis. Inter. Sci. Publishers, Inc., New York.

Reganold, J. P. (1988). Comparison of soil properties as influenced by organic and conventional farming systems. American Journal of Alternative Agriculture, 3(4), 144-155.

Relf, D.,McDoniel,A., and Tech, V., (2002): Fertilising the vegetable garden. http://www.indiaagronet.com/indiaagronet/Manu ers_fer tilizers/contents/inorganic_fertilizers.htm
Roe, N. E. and Comforth, G.C. (1997). Yield effects and economic comparison using fresh or composted dairy manure amendments on double-cropped vegetables. Hort Science, 32(3) 462-463.

Seran, T. H., Srikrishnah, S., and Ahamed, M. M. Z. (2010). Effect of different levels of inorganic fertilizers and compost as basal application on the growth and yield of onion (Allium cepa L.). Journal of Agricultural Sciences, 5(2), 64-70

Shebl ,E.F. (1995). Studies on the effect of some mineral nutrients on yield and quality of cantaloupe ( Cucumis melo var. cantaloupensis ,L).M.Sc. Thesis, Fac. Agric., Mansoura Univ., Egypt

Smil, V. (2001). Enriching the earth: fritz haber, Carl Bosch, and the Transformation of World Food Production. Cambridge, USA: The MIT Press

Tindall, H.D. (1968). Commercial vegetable growing. Tropical hand book series, Oxford University Press. Oxford. pp 635-637..

Vernon, G. (1999). Sustainable vegetable Production from start-up to market. Cornell Univesity. Ithaca, New York

Worthington, V. (1998). Effect of agricultural methods on nutritional quality: a comparison of organic with conventional crops. Alternative Therapies Health Med., 4 (1): 58-69

Worthington, V. (2001). Nutritional quality of organic versus conventional fruits, vegetables and grains. J. Alternative Complent. Med., 7: 161-173.

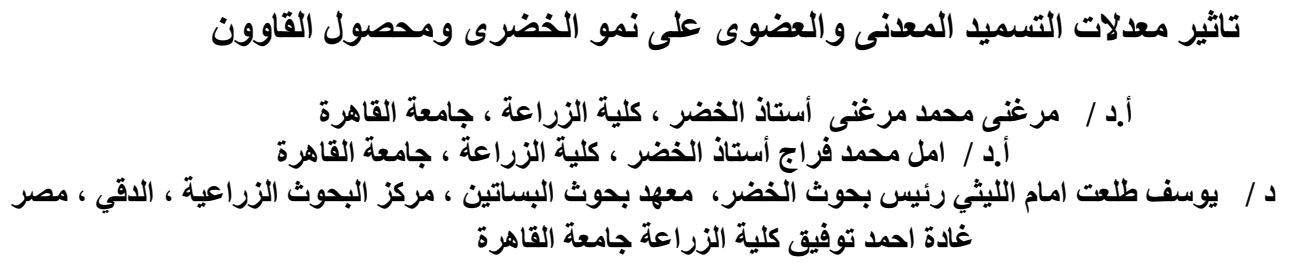

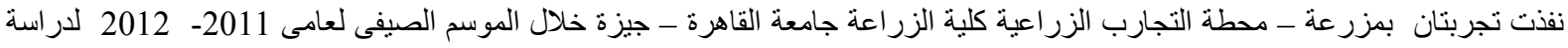

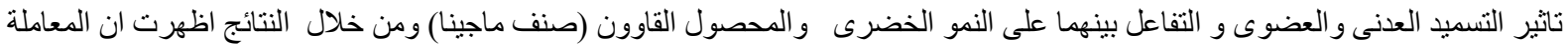

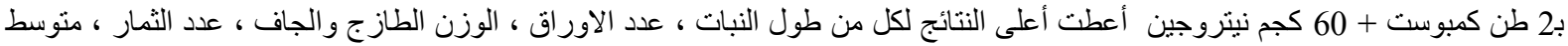

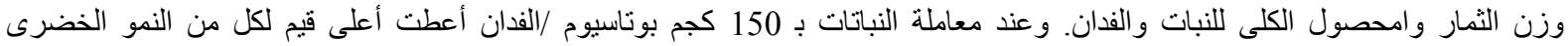

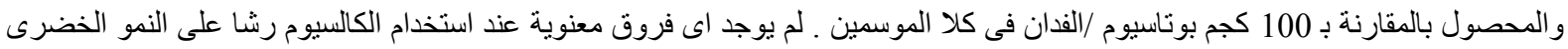

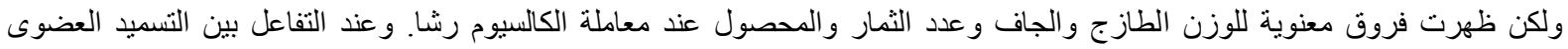

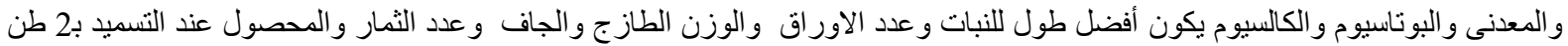

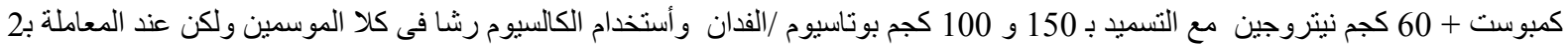

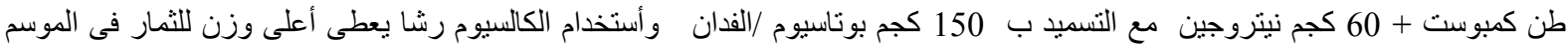

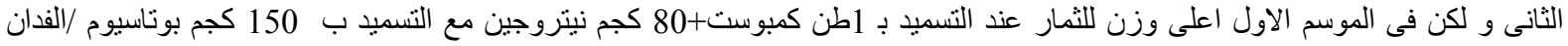
و وأستخدام الكالسيوم رشا. الكلمات المفتاحية: القاوون ،التسميد العضوى و المعدنى، نمو الخضرى، المحصول. 